OPEN ACCESS

Edited by:

Shouju Wang

Nanjing Medical University, China

Reviewed by:

Rajendra Kumar Singh,

Institute of Tissue Regeneration

Engineering (ITREN), South Korea

PaYaM ZarrinTaj,

Oklahoma State University,

United States

*Correspondence:

Dongqing Wang

wangdongqing71@163.com

Specialty section:

This article was submitted to Biomaterials,

a section of the journal

Frontiers in Bioengineering and Biotechnology

Received: 30 December 2020 Accepted: 04 March 2021

Published: 13 April 2021

Citation:

Gao Q, Zhang J, Gao J, Zhang Z, Zhu H and Wang D (2021) Gold Nanoparticles in Cancer Theranostics. Front. Bioeng. Biotechnol. 9:647905. doi: 10.3389/fbioe.2021.647905

\section{Gold Nanoparticles in Cancer Theranostics}

\author{
Qinyue Gao, Jingjing Zhang, Jie Gao, Zhengyang Zhang, Haitao Zhu and \\ Dongqing Wang*
}

Department of Radiology, Affiliated Hospital of Jiangsu University, Zhenijang, China

Conventional cancer treatments, such as surgical resection, radiotherapy, and chemotherapy, have achieved significant progress in cancer therapy. Nevertheless, some limitations (such as toxic side effects) are still existing for conventional therapies, which motivate efforts toward developing novel theranostic avenues. Owning many merits such as easy surface modification, unique optical properties, and high biocompatibility, gold nanoparticles (AuNPs and GNPs) have been engineered to serve as targeted delivery vehicles, molecular probes, sensors, and so on. Their small size and surface characteristics enable them to extravasate and access the tumor microenvironment (TME), which is a promising solution to realize highly effective treatments. Moreover, stimuli-responsive properties (respond to hypoxia and acidic $\mathrm{pH}$ ) of nanoparticles to TME enable GNPs' unrivaled control for effective transport of therapeutic cargos. In this review article, we primarily introduce the basic properties of GNPs, further discuss the recent progress in gold nanoparticles for cancer theranostics, with an additional concern about TME stimuli-responsive studies.

Keywords: optical property, nanomedicine, cancer theranostics, tumor microenvironment, gold nanoparticle

\section{INTRODUCTION}

The researches of cancer treatments have been developing at an extremely rapid pace to support the personalized demands for better precision therapeutics. Currently, cancer treatment strategies for solid tumors include surgery, radiation therapy, chemotherapy, immunotherapy, and a combination of some or all of these mentioned approaches (Nicolson et al., 2020). Unsatisfactory antitumor performance and some severe side effects have always been a confusing problem in clinical treatments. The intrinsic limits of conventional cancer therapies prompt the increasing interests in the applying of nanotechnology in the diagnosis and therapy assessments for cancer, which fight cancer cells more accurately with fewer potential side effects. Nanotechnology (Wilhelm et al., 2019) as new treatment modalities, can greatly enhance the precise anticancer efficacy, showing promising potentials in clinical translations. Gold nanoparticles (GNPs) are of increasing interest because their excellent biocompatibility and physicochemical properties (Chen et al., 2016) can support multiple functions. Since the first scientific article attributing the red color to the colloidal nature of Au-based NPs reported by Faraday in 1857, AuNPs at present have been engineered as stable nanocargoes (Wang C. et al., 2018), metal catalysts (Vidal et al., 2018), organic photosensitizers (PS) (Li S. et al., 2019), or to directly produce ROS 
and heat under near-infrared light irradiation (Mangadlao et al., 2018), which promise biological friendly nanoparticles in the medical treatment and diagnostics.

However, ideal antitumor functions of GNPs need to be favored by the tumor's surrounding environment. The tumor microenvironment (TME) dictates tumor progression, regulates cancer cell behaviors, and persistently provides nutrition for cancer growth and metastasis (Figure 1). Several common features of TME, such as the vascular abnormalities, the extracellular matrix (hypertonicity, hypoxia, and $\mathrm{pH}$ ), and the immune response of TME are quite different from normal tissues (Peng et al., 2019). These are the reasons that TME-triggered theranostic gold nanoparticles could offer promising perspectives to achieve tumor-specific detection and therapy (Table 2). In this review, we aim to illustrate the basic properties of GNPs and current researches on the development of GNPs including the interactions between nanomaterials and the TME.

\section{PROPERTIES OF GOLD NANOPARTICLES}

\section{Localized Surface Plasmon Resonance}

Gold nanoparticles spark broad interest due to their unique optical performance (Wilson et al., 2020). The external specific wavelength laser excites a collective and coherent oscillate of the conduction electrons near the gold nanoparticle's surface, and this oscillate is in resonance with the incident light frequency leading to scattering peaks and spectral absorption as well as local field enhancements (Figure 2A), which is the important optical property of GNPs-localized surface plasmon resonance (LSPR) (Zhou et al., 2015; Ramos et al., 2018). Many studies have confirmed that LSPR of GNPs is exquisitely sensitive to size (Takano et al., 2018), material geometry (Rebello Sousa Dias and Leite, 2019), dimension, and dielectric properties (Agrawal et al., 2018) of the surrounding media (Figure 2B). For instance, AuNPs have been produced in different shapes, such as the most common nanospheres, nanoshells, nanorods (NRs), nanostars, nanocages, and core-shell structures (Table 1). Changing the structural geometry of gold nanoparticles can readily and precisely tune the LSPR from visible to the nearinfrared (NIR) region without giving up linewidth (Tan et al., 2018). As an important factor that may affect LSPR, the size of gold nanoparticles is multiple. The increased rod aspect ratios of gold nanorods (AuNRs) show a significant peak shift to the near-infrared region (He et al., 2020). Distinctly from AuNRs, gold nanoshells (AuNSs) are highly sensitive to the shell thicknesses, which similarly change their plasmonic properties (Ogunyankin et al., 2018). Going beyond the conventional impact of LSPR factors-the shape and size of GNPs, new synthetic control methods are also the affecting factor of LSPR and require expanding the freedom in structural and functional designs. Yuhua Feng's group synthetically controlled the non-wetting growth of homometallic nanostructure in two new dimensions (the number and emerging shapes of the Au islands), obtaining a series of Au-on-AuNR hybrid structures, and effectively and continuously tuned their LSPR within visible-NIR spectral range, making them excellent candidates for photothermal therapy and photoacoustic imaging agents (Jia J. et al., 2020). The electric field of LSPR is also highly dependent on the dielectric property of the supporting substrates because the interaction between the gold nanostructures and the surrounding medium may change the refractive index of the GNPs (Bhalla et al., 2019), and when the local refractive index of the NPs increases, this always causes the decreases in LSPR frequency; thus, the sensitivity of the LSPR to the refractive index of the surrounding substrates can also be used for detecting dynamic gold nanostructures and the ambient chemical reactions (Ogunyankin et al., 2018) in the changes in LSPR scattering.

Besides, LSPR is applied in many imaging techniques, such that dark-field microscopy (DFM) and two-photon luminescence (TPL) primarily use the light scattering property of GNPs, while photoacoustic imaging often applies the optical absorption of GNPs. Two-photon luminescence imaging, through scanning a focused laser beam over the target tissues, has been used to visualize distributions of localized surface plasmons in nanoparticles within the NIR region. This TPL is extremely sensitive to the highly enhanced electro-magnetic fields connected with plasmonic excitations in gold nanostructures (Durr et al., 2007). Consequently, the collective oscillations of the electrons in the gold nanoparticles generate strong TPL signal enhancements. In addition, gold nanorods, as appealing TPL imaging substrates, can strongly scatter to detect cancer cells under excitation in the near-infrared region where biological tissues exhibit slight extinction coefficients (Zhang W. et al., 2018). TPL imaging from gold-nanorod-labeled cancer cells could be acquired by using less than 60 times the laser light excitation power required for two-photon auto-fluorescence (TPAF) imaging of unlabeled cancer cells, consistent to three orders of magnitude amplified in an emitted signal for equal excitation intensity (Durr et al., 2007).

\section{Near-Infrared Region}

Due to the scattering and absorption of light by biological tissues, UV and visible light own a limited penetration depth into and then propagation out of tissues not taking deeptissue imaging. Near-infrared light can penetrate more efficiently into tissues such as skin and blood than UV and visible light because the main absorbers of visible and infrared lightwater and hemoglobin have their lowest absorption coefficient and scattering of excitation emission photons in the nearinfrared region (Shanmugam et al., 2014), thus providing maximum laser penetration in the tissue and minimizing the autofluorescence of the non-target part (Smith et al., 2009). The near-infrared window is divided into two distinct spectral ranges (Figure 3A): NIR-I (traditional NIR window, $700 \sim 900 \mathrm{~nm}$ ) and NIR-II windows (the second NIR window, $1,000-1,700 \mathrm{~nm}$ ) (Ji et al., 2020). Chen et al. (2019) synthesized miniaturized gold nanorods which are 5-11 times smaller than the regular size under nanosecond laser irradiation. Small AuNRs absorbing at $1,064 \mathrm{~nm}$, are used to photoacoustic molecular imaging applications that significantly enhanced photoacoustic signal of overall cancer, and lower background noise of endogenous absorbers in tissues. With the advancement of NIR fluorophores 

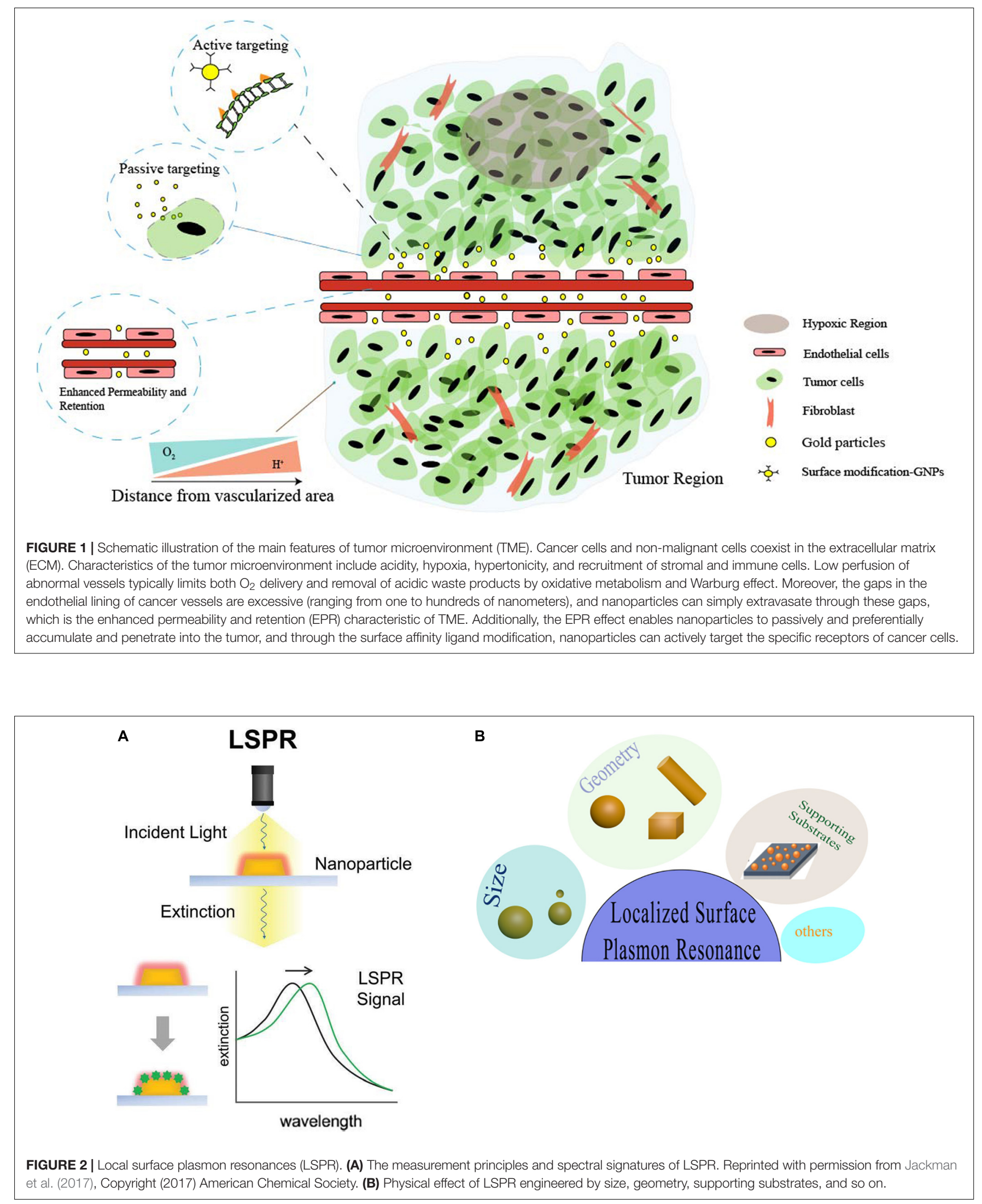
TABLE 1 | Summary of various gold nanostructures.

\begin{tabular}{|c|c|c|c|c|}
\hline Nanostructure & Synthesis methods & Characterization & Shortcomings & References \\
\hline Gold nanotube & Template synthesis mechanism & $\begin{array}{l}\text { Tunable plasmonic resonances } \\
\text { in NIR region; larger active } \\
\text { areas and more electroactive } \\
\text { sites for immobilizing } \\
\text { biomolecules; high scattering } \\
\text { contrast; } \\
\text { high sensitivity }\end{array}$ & $\begin{array}{l}\text { Cytotoxic potential due to } \\
\text { chemical } \\
\text { synthesis }\end{array}$ & Liu et al., 2020 \\
\hline Gold nanorod & $\begin{array}{l}\text { Template synthesis mechanism; } \\
\text { seed-mediated protocols; } \\
\text { seedless methods; } \\
\text { the green synthesis }\end{array}$ & $\begin{array}{l}\text { Two typical LSPR peaks with } \\
\text { tunable plasmonic resonances } \\
\text { in NIR region; } \\
\text { high sensitivity; } \\
\text { easy surface functionalization; } \\
\text { antimicrobial activity mode; } \\
\text { strong magnitude of saturable } \\
\text { absorption }\end{array}$ & $\begin{array}{l}\text { Cytotoxicity, accumulation in } \\
\text { liver in vivo, poor photostability. }\end{array}$ & $\begin{array}{l}\text { Alkilany et al., 2012; Ma et al., } \\
2017\end{array}$ \\
\hline Gold nanocluster & $\begin{array}{l}\text { Chemical etching; } \\
\text { Chemical reduction; } \\
\text { Sonochemical synthesis; } \\
\text { Electrochemical synthesis; } \\
\text { Microwave-assisted synthesis }\end{array}$ & $\begin{array}{l}\text { Tunable plasmonic resonances } \\
\text { in NIR region; ultra-small size; } \\
\text { strong photoluminescence; } \\
\text { sturdy photostability; fast renal } \\
\text { elimination; excellent } \\
\text { biocompatibility; } \\
\text { extremely large surface area }\end{array}$ & Cell apoptosis & $\begin{array}{l}\text { Zhang Q. et al., 2018; Zhao } \\
\text { et al., } 2014\end{array}$ \\
\hline Gold nanostar & $\begin{array}{l}\text { Seed-mediated method; } \\
\text { seedless synthesis method; } \\
\text { chemical reduction of gold salt method; } \\
\text { surfactant-free method }\end{array}$ & $\begin{array}{l}\text { Multiple plasmon resonances; } \\
\text { low toxicity; } \\
\text { high antibacterial activity; } \\
\text { great surface plasmon } \\
\text { resonance; } \\
\text { easy surface functionalization }\end{array}$ & Cytotoxicity & Mousavi et al., 2020 \\
\hline Gold nanocage & Galvanic replacement reaction & $\begin{array}{l}\text { Enhanced absorption in NIR } \\
\text { regions; } \\
\text { hollow interiors and porous } \\
\text { walls; } \\
\text { extraordinarily large scattering } \\
\text { and absorption cross sections; } \\
\text { easy surface functionalization }\end{array}$ & Melting point at a power density & Xia et al., 2011 \\
\hline Gold nanoshell & $\begin{array}{l}\text { Gold nanoshells on } \mathrm{SiO}_{2} \text { core: } \\
\text { surfactant-assisted seeding method; } \\
\text { single step deposition-precipitation (DP) } \\
\text { seeding; sandwiched gold seeded shell } \\
\text { Gold nanoshells on a polymer core: } \\
\text { solvent-assisted approach; combined } \\
\text { swelling-heteroaggregation; gold colloid } \\
\text { seeding } \\
\text { Hollow gold nanoshells: sacrificial } \\
\text { template method }\end{array}$ & $\begin{array}{l}\text { Tunable plasmonic resonances } \\
\text { in NIR regions; } \\
\text { highly effective for PTT and } \\
\text { SERS }\end{array}$ & Weak non-linear response & Ma et al., 2017 \\
\hline Gold nanosphere & $\begin{array}{l}\text { Wet chemistry method; } \\
\text { seed-mediated growth }\end{array}$ & $\begin{array}{l}\text { Single LSPR peak; the smallest } \\
\text { specific surface area; high } \\
\text { cellular internalization; highest } \\
\text { colloidal stability; easy and } \\
\text { available synthetic } \\
\text { methods; easy surface } \\
\text { functionalization }\end{array}$ & $\begin{array}{l}\text { Cytotoxicity, accumulation in } \\
\text { different organs in vivo }\end{array}$ & Wozniak et al., 2017 \\
\hline
\end{tabular}

LSPR, localized surface plasmon resonance; NIR, near-infrared region; SERS, surface-enhanced Raman spectroscopy; PTT, photothermal therapy.

and adequate sensitive photon detectors, the NIR window can expand to the longer wavelengths with further reduced scattering, deeper penetration and high-spatial resolution in molecular imaging (Ding et al., 2018). NIR-II fluorescence imaging in vivo is a much newer field of research. Compared with traditional imaging approaches using the shorter emission wavelength windows, NIR-II has deeper tissue penetration capability and higher signal-background ratio (SBR). However, due to the poor aqueous solubility, generally low quantum yields, and lack of suitable molecules with bandgaps, the longer fluorescence emission wavelength in small-molecule organic dyes is difficult in the NIR-II window. Fortunately, NIR-I fluorophore indocyanine green (ICG) has been explored for NIR-II imaging of cancer in clinic, and the advantages of NIR-II imaging for tumor imaging 
TABLE 2 | The applications of gold nanostructure in tumor environment.

\begin{tabular}{|c|c|c|c|c|}
\hline Main affections on TME & Composite & $\begin{array}{l}\text { Therapeutic } \\
\text { modality of GNPs }\end{array}$ & Wavelength & References \\
\hline & Au@BSA-NHA & CT imaging contrast & & Shi et al., 2016 \\
\hline $\begin{array}{l}\text { Hypoxia-sensitive } \\
\text { nanoprobe }\end{array}$ & $\begin{array}{l}\text { Anaerobic bacteria-GNP } \\
\text { conjugates }\end{array}$ & Photothermal therapy & NIR-II & Luo et al., 2016 \\
\hline \multirow[t]{3}{*}{ Alleviating hypoxia } & $\begin{array}{l}\text { Au-hemoglobin loaded } \\
\text { platelet }\end{array}$ & Radiosensitizers & NIR-II & You et al., 2020 \\
\hline & $\begin{array}{l}\text { ICG-PtMGs(Pt- } \\
\text { MOF@GNSs)@HGd }\end{array}$ & $\begin{array}{l}\text { Photothermal therapy } \\
\text { CT and MRI imaging }\end{array}$ & NIR-II & You et al., 2020 \\
\hline & Gold cube-in-cube core & Photothermal therapy & NIR-I & Zhang et al., 2019 \\
\hline $\begin{array}{l}\text { Glutathione-responsive } \\
\text { drug delivery }\end{array}$ & $\mathrm{SiO}_{2} @ \mathrm{Au} @ \mathrm{MnO}_{2}-\mathrm{DOX} / \mathrm{Apt}$ & Photothermal therapy & $\begin{array}{l}\text { UV-vis spectroscopy } \\
\text { and NIR-I }\end{array}$ & Zhang T. T. et al., 2018 \\
\hline pH-activatable nanoprobe & GNPs-CKL-FA & CT contrast agent & NIR-I & Tang et al., 2019 \\
\hline $\begin{array}{l}\text { pH-responsive drug } \\
\text { delivery }\end{array}$ & Gold nanostar@ZIF-8 & $\begin{array}{l}\text { Drug carriers } \\
\text { photothermal }\end{array}$ & NIR-II & Deng et al., 2019 \\
\hline $\begin{array}{l}\text { Cathepsin B-responsive } \\
\text { nanomedicine }\end{array}$ & $\begin{array}{l}\text { Multifunctional } \\
\text { peptide-coated ultrasmall } \\
\text { gold nanoparticles }\end{array}$ & $\begin{array}{l}\text { Radio-sensitizing } \\
\text { cytotoxicity }\end{array}$ & X-ray & Ding et al., 2020 \\
\hline $\begin{array}{l}\text { Inactivation of } \\
\text { cancer-associated } \\
\text { fibroblasts (CAF) }\end{array}$ & Gold nanoparticles & Inhibit CAF & & Zhang et al., 2021 \\
\hline SERS analysis of CAF & MoS2-Au & SERS-active probe & NIR-II & Li et al., 2020b \\
\hline Normalizing tumor vessels & AuNPs-A\&C & Drug delivery & Photoacoustic & Xiao et al., 2017 \\
\hline
\end{tabular}

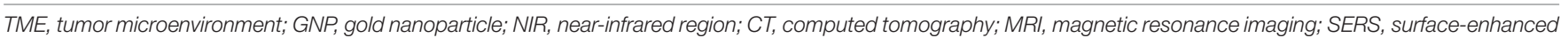
Raman spectroscopy.

and image-guided therapy is worth studying (Zhu et al., 2018). Li et al. (2020a) discovered that $\mathrm{Au}_{25}(\mathrm{SG})_{18}$ was a highly promising bone-targeted NIR-II probe. Dynamic NIR-II imaging of the whole body in mice receiving $\mathrm{Au}_{25}(\mathrm{SG})_{18}$ displayed all dorsal bone structures ranging from cranial to spin with high resolution and contrast, and even clearly defined the pelvis and femur (Li et al., 2020a).

\section{Surface-Enhanced Raman Spectroscopy}

The surface-enhanced Raman spectroscopy (SERS) has developed from an esoteric physical phenomenon to an effective analytical measurement technique (Lindquist et al., 2019). Once an incident light hits a sample, the major proportion of the scattered light from the sample have the same frequency, representing the Rayleigh scattering effect. Nonetheless, about a millionth of photons is inelastically scattered from the sample with an energy unlike the incident light energy, corresponding to the Raman scattering effect, which is unique for every molecule providing precise spectral fingerprint of the samples, and the difference between Raman scatter and the incident light is proportional to the molecular vibrational states (Liebel et al., 2020). When molecules are located near a plasmonic material, the Raman signals can be significantly amplificated, leading to the SERS process (Ding et al., 2017; Zong et al., 2018; Figure 3B). There are at least three mechanisms in relation to the enhancement factors: the dominating factor first mentioned is electromagnetic (EM) enhancement induced by the local surface plasmon resonance, which is irradiated by a certain wavelength range light resulting in collective oscillation of the conduction electrons in the nanostructured metal substrate. The second is the chemical enhancement (CM) under resonance related to the charge transfer between the sample molecules and the metal nanostructure substrate, but it is molecule specific, and compared with EM enhancement, it presents a lower enhancement factor. The third possibility is the resonances within the molecule itself (Zong et al., 2018). In vivo, Raman signals are inherently weak for imaging and drug location tracking. Surface-enhanced Raman spectroscopy can gain the dramatic amplification of the Raman scattering efficiencies when molecules are grafted on the surfaces of GNPs and take the superiorities in the gold nanostructure plasmon features. After AuNPs efficiently target the cell outer membrane proteins, using SERS from the surface of AuNPs can quantify the local $\mathrm{pH}$ induced by $\mathrm{H}^{+}$extrusion in MKN28 gastric cancer cells and HepG2 liver cancer cells, visualizing the dynamics of proton exchange in cells (Puppulin et al., 2018). GNPs coated by target-protein-immobilized substrate in direct SERS-based immunoassay (SERSIA) closely contact target proteins, obtaining the SERS signal of the target protein signal and proving the feasibility of protein imaging at a micrometer range. This detection protein sensitivity was comparable to the ELISA, and the correlation between GNPs and target protein was well matched. Through this method, SERSIA can reliably estimate the limitation of the protein level at a sub-picomolar detection (Shin et al., 2020). As an alternative method to traditional fluorescence-based spectroscopy, SERS not only owns ultrasensitive multiplexing capability (Samanta et al., 2014), non-photobleaching feature (Zhang J. et al., 2018), sub-picomolar level sensitivity, and high signal-to-noise ratio (Amendola, 2019) but also enables ultrasensitive detections of molecular vibrations even at the standard for the high 


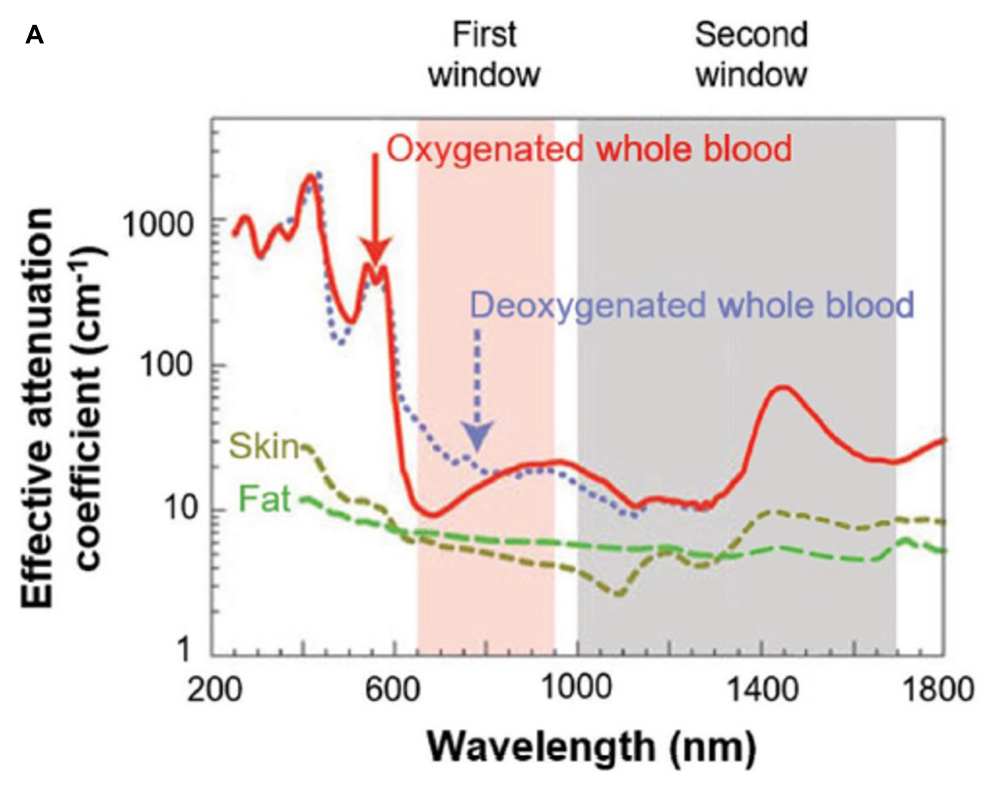

B

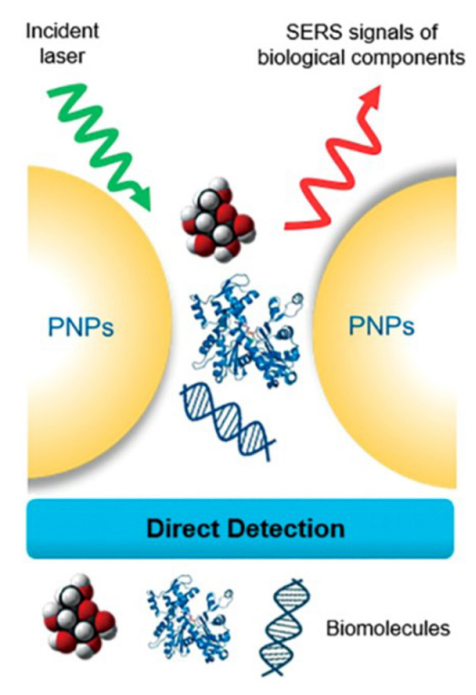

FIGURE 3 | Schematics of (A) near-infrared region, (B) surface-enhanced Raman spectroscopy. (A) The absorption spectrum of biological tissues spanning from 200 to $1,800 \mathrm{~nm}$. The absorption and scattering of whole blood, skin, and fat are significantly reduced in either NIR-I (700-900 nm, shaded in pink) or NIR-II (1,000-1,700 nm, shaded in gray) window. Reprinted with permission from Kenry et al. (2018), Copyright (2018) John Wiley and Sons. (B) The direct SERS detection. Reprinted (adapted) with permission from Zong et al. (2018), Copyright (2018) American Chemical Society.

structural selectivity in a single molecule. SERS sensing with a flow-through scheme has been applied in DNA and protein analysis. Gold nanourchins (AuNUs) have many sharp tips. And the electromagnetic field can be concentrated at the sharp tip in the anisotropic structure that can act as an excellent "hot spot," resulting in a dominant contribution to the SERS intensity. When the SERS platform was utilized to detect a single molecule, nucleotides are mixed in the AuNUs solution to allow an average submonolayer coverage on the surface of the AuNUs. Once trapped, plasmonic hot spots are created by coupling the sidewall of the nanohole and the sharp tips of the AuNUs, which exhibit an intense, confined electromagnetic field for SERS detection of the DNA already adsorbed on the tips. Through applying electric potentials, nucleotides can stay in the hot spots sufficiently long to achieve single-molecule detections (Huang et al., 2019).

\section{Light-Triggered Modalities-PDT and PTT Photodynamic Therapy}

Photodynamic therapy (PDT) is an emerging and minimally invasive treatment modality with a spatiotemporal selectivity. It employs photosensitizer molecules to absorb the excitation light of a certain wavelength and generate reactive oxygen species (ROS) to selectively damage tumor tissues in situ. Three essential factors in PDT are non-ionizing light providing energy for photodynamic reaction, a photosensitizer (PS) harvesting this light energy and participating in the reaction, and molecular oxygen-producing ROS upon electron transfer from excited photosensitizers (Zhou et al., 2016). When the photosensitizer is irradiated to a photon of light, it becomes activated from its ground state (singlet state) to a short-lived excited singlet state and can convert its energy to emit fluorescence or internal transformation into heat, which is the mechanism for quantifying the amount of photosensitizer in cells or tissues and fluorescence imaging in vivo monitoring the distribution and pharmacokinetics of the PS. The short-lived excited singlet state PS may also experience the process known as intersystem crossing that the spin of its excited electron inverts to acquire the relatively long-lived (microseconds) excited triplet state (Dolmans et al., 2003). The excited triplet can react with a substrate directly, such as the cell membrane or a molecule, and transfer an electron to form a radical atom. Then these radicals interact with ground-state molecular oxygen to generate oxygenated products including hydroxyl radicals, superoxide anion radicals, hydrogen peroxides, and so on (type I reaction). Alternatively, the energy of the excited triplet can be transferred directly to oxygen, to form singlet oxygen-a highly ROS (type II reaction) (Chatterjee et al., 2008; Figure 4A). Reactive oxygen species (ROS), broadly defined as chemically reactive oxygencontaining small molecules, include the superoxide anion $\left(\mathrm{O}_{2}{ }^{-}\right)$, hydroxyl $(\mathrm{OH} \cdot)$ radicals, hydrogen peroxide $\left(\mathrm{H}_{2} \mathrm{O}_{2}\right)$, and singlet oxygen (Sies and Jones, 2020). Singlet oxygen is the most prevalent reactive oxygen species produced upon the activation of photosensitizers to induce irreversible damage to tumor cells. Upon extrapolation to an $\mathrm{H}_{2} \mathrm{O}$-containing cell, a singlet oxygen has a lifetime of $23.5 \mu$ s (Hatz et al., 2007).

Gold nanoparticles generally display excellent photostability and strong two-photon photoluminescence, owning a high potential to be applied in two-photon excitation (TPE) PDT. The TPE occurs when the sum of the absorption of two lowerenergy photons equals the energy of the band gap of the PSs, providing a potential for deeper tissue penetration and lower 

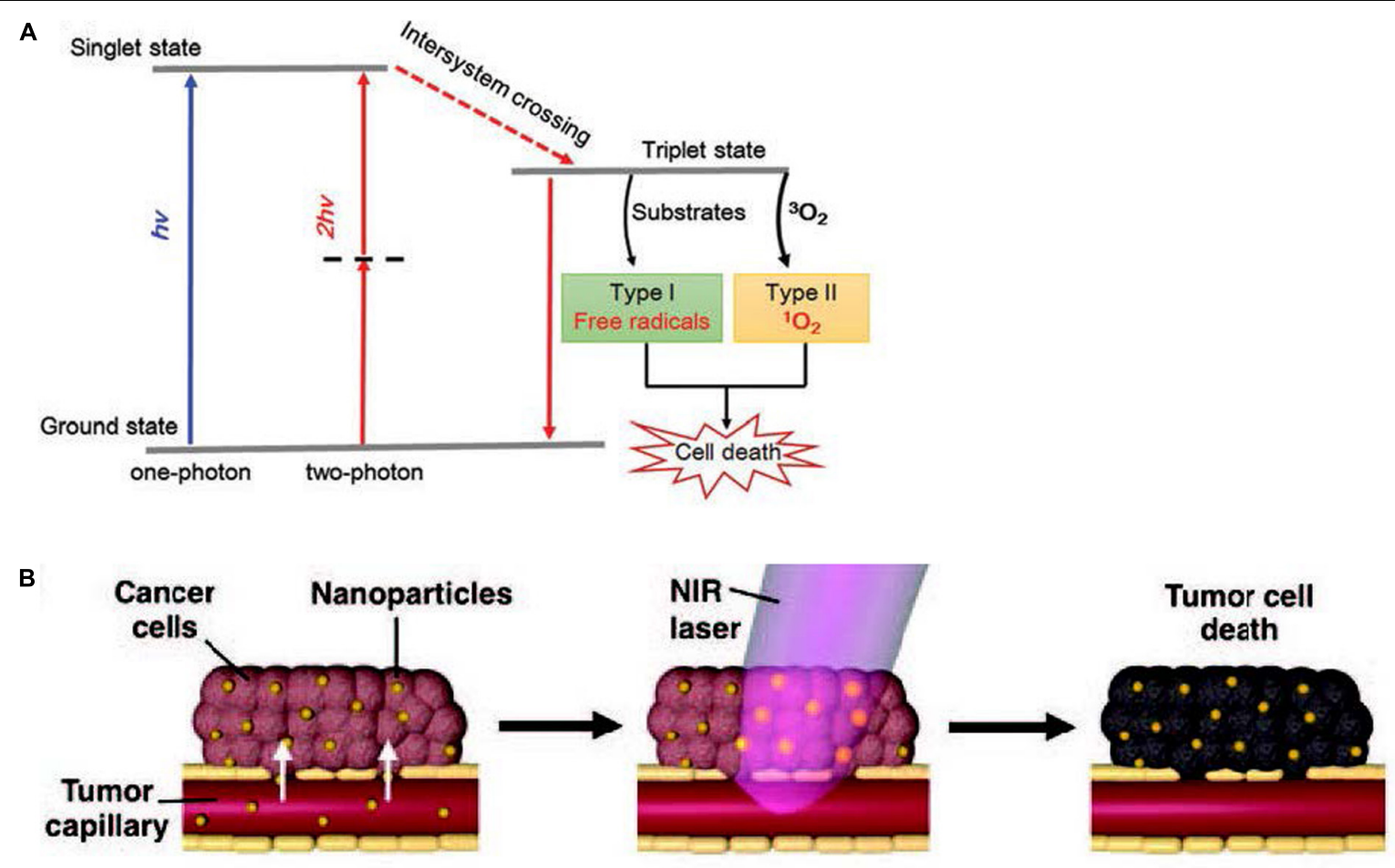

FIGURE 4 | (A) Schematic illustration of type I and type II mechanisms in photodynamic therapy (PDT). Reprinted with permission from Shen et al., 2016, Copyright (2016) Royal Society of Chemistry. (B) Schematics demonstrating photothermal therapy. Reprinted with permission from Riley and Day (2017), Copyright (2017) John Wiley and Sons.

photo-bleaching of PS molecules (Shen et al., 2016). Furthermore, the TPPL properties of GNPs are strongly correlated with the geometry. The two-photon action cross sections of single gold nanospheres $(\sim 83 \mathrm{GM})$, nanocubes $(\sim 500 \mathrm{GM})$, nanotriangles $\left(\sim 1.5 \times 10^{3} \mathrm{GM}\right)$, nanorod $\left(\sim 4.2 \times 10^{4} \mathrm{GM}\right)$, and nanobranches $\left(\sim 4.0 \times 10^{6} \mathrm{GM}\right)$ were dramatically different. Laser-induced melting experiments found that the sharp tips of these nanobranches were closely correlated with observed strong TPPL (Gao et al., 2014).

\section{Photothermal Therapy}

The vascular system in tumor undergoes agglomeration, distortion, and expansion, resulting in its failure in taking heat away quickly like the normal vascular system does. Owing to inadequate bloodstream and oxygen transport through the newly formed immature tumor vascular system, tumor cells reside in a nutrient-deprived and acidotic milieu that confers them with greater sensitivity to heat (Wang et al., 2016). Meanwhile, malignant cells have impaired heat shock protection mechanisms, so the cytotoxicity profile of mild hyperthermia is usually very good. Temperature rises rapidly above the threshold value of $42-45^{\circ} \mathrm{C}$ [as biological cells begin to die from hyperthermia when the temperature reaches up to $43^{\circ} \mathrm{C}$, on the other hand, high doses of heating $\left(>50^{\circ} \mathrm{C}\right)$ induce necrosis resulting in inflammation and is harmful to the neighboring normal cells] for 15-60 $\mathrm{min}$ at the intracellular location target sites, leading to ablate cancer cells and consequently to cell death (Figure 4B;
Rengan et al., 2015). Conventional methods have poor therapeutic effect on deep-seated tumor, and the heating distribution is always not well controlled, such as hyperthermia produced by microwaves or heat applicators. More importantly, these methods are only macroscopically confirmed to the tumor area but not especially at the cellular level (Vo-Dinh et al., 2013). The investigation of nanoparticles as conduits for generating hyperthermia is tumor focused, minimally invasive, and uniform. Numerous nanomaterials with strong NIR absorption ability present great potential in photothermal treatment, achieving encouraging therapeutic effect in vivo.

AuNPs usually exhibit strong absorbance in the NIR region with a much higher absorption cross-section compared with the small organic dyes, whose peak could be changed by the morphology and aggregation state of AuNPs (Goncalves et al., 2020). When the administered gold nanoparticles transmitted in the blood flowed across the tumor vessels under NIR laser irradiation, they convert the effective delivered optical energy into localized heat, causing the tumor temperature to increase (Vo-Dinh et al., 2013; Cheng et al., 2014). The direction of heat loss in GNPs is from inside out reducing damage to normal tissues comparable with other means of external hyperthermia, and they make the steady temperature increase in the tumor (Moy and Tunnell, 2017). Gold nanorods are one of the most studied GNPs in PTT, which have excellent photothermal conversion efficiency and aspect ratio-dependent absorption peak positions. The anisotropic gold nanorod shape splits the LSPR 
into two plasmon modes (Vigderman et al., 2012): one is a weak short-wavelength band around $520 \mathrm{~nm}$ in the visible region formed by the transverse electronic oscillation, which is relatively unaffected by nanorod size; the other is a strong long-wavelength band in the near-infrared region from the oscillation of electrons along the long axis. AuNRs can provide a large electric field, and the enhancements are primarily located in the regions at the two ends of the nanorods (Chen et al., 2013). Combined with various aspect ratios (the ratio between the length and the width), these properties enable GNRs to become attractive candidates for exploitations in PTT.

\section{STABILIZATION, BIODISTRIBUTION, BIOCOMPATIBILITY IN VIVO}

Gold nanoparticles can either be directly intravenously injected into the bloodstream or interact with the primary tissue barriers and then translocate into the bloodstream. When GNPs are exposed to blood or biological fluids, proteins are adsorbed onto the GNP surface at first for a few minutes forming a socalled protein corona (PC), which may be well organized by irreversible interactions forming the long-lived hard corona, or through loose "protein-protein" associations forming a rapidly transient layer of biomolecules, providing a soft corona (Feliu et al., 2016). The protein corona cannot only induce steric stabilization and reduce non-specific cellular uptake but also promote the destabilization effect of GNPs by impacting proteinmediated bridging, changing surface charge compensation and inhomogeneity onto the GNPs (Charbgoo et al., 2018). Notably, this kind of proteins forming the outer layer affect the suborgan biodistribution of AuNPs. Generally, the fate of the GNPs in vivo commonly depends on physicochemical properties, mechanical properties, and surface charge. Therefore, this section reviews the mechanical properties of GNPs in blood circulation, biodistribution, and tumor accumulation.

\section{Stabilization}

The stabilization of the fabricated gold nanoparticles demonstrating the ability to protect GNPs from aggregation or dissolution in colloidal dispersion is crucial for preservation of the desired plasmonic performance. There are various strategies used to achieve stabilization (Figure 5). The cetyltrimethylammonium bromide (CTAB) as a bilayer via electrostatic interactions absorbed on the gold surface has been predominately utilized as a shape controller and a stabilizer. A bilayer structure around the GNRs consisting of CTAB increases colloidal stability in aqueous solution. CTAB coordinating with another mild reducing agent could obtain anisotropic AuNPs, which is vital for potential applications in SERS. However, its high cytotoxicity is a major concern, and in a biological setting, the CTAB layer must either be exchanged or encapsulated (Shi et al., 2021). Polyethylene glycol (PEG) (Huckaby and Lai, 2018) is another commonly used stabilizer prolonging the blood circulation time in vivo. Clinically, PEG is approved for intravenous application (Rafiei and Haddadi, 2017). PEG chains are hydrophilic and non-toxic polymers having a flexible nature, which assemble into dense palisades of tethered chains to achieve unique properties (D'Souza and Shegokar, 2016). The dense PEG shell endows the AuNPs with a stealth character to avoid non-specific recognition by the immune system guaranteeing the biocompatibility of AuNPs' effective accumulation in the tumor area based on enhanced permeability and retention (Chan et al., 2020). Apart from the optimization of PEG, considering the interaction and the stabilization of GNPs in blood plasma, bovine serum albumin (BSA) as the most abundant plasma protein holds great promise for biomedical applications. The BSA-coated AuNPs showed high colloidal stability and biocompatibility with no hemolytic response, significantly in contrast to CTAB-capped AuNPs (Tebbe et al., 2015). Nevertheless, BSA has been mainly used as a template and a secondary stabilizing agent on account of the structural complexities and difficulties in controlling during modification. Additionally, biomolecules like DNA have been broadly used as a ligand to provide steric stabilization and high electrostatic repulsion forces to GNPs due to strong negative charge on the phosphate backbone (Liu and Liedl, 2018). In general, these strategy stabilities of GNPs are mainly assayed in vitro, and firmly grafted stabilizers around gold nanoparticles may degrade when injected in vivo leading to the dramatic changes in physicochemical properties and integrity of GNPs (Kreyling et al., 2015). Therefore, future research will likely encompass the enhancement of the stabilities of the AuNPs in vivo.

\section{Blood Circulation}

Once GNPs are administered into the blood, these nanoparticles are exposed to the dynamic environment of the bloodstream. Flow rate is a fundamental property that influences the transport of gold nanoparticle and interaction with endothelial cells. The decreased uptake of nanoparticles by the endothelial cells occurs in increased flow rates and eventually reduces the amounts of GNPs entering the tumor region through trans-endothelial processes (Chen et al., 2020). As mentioned above, despite PEG ensuring the blood circulation of GNPs, the length of the PEG is essential for the circulation times of NPs. Lipka et al. (2010) found that $10-\mathrm{kDa}$ PEG chains have a prolonged blood circulation time than non-PEGylated AuNPs and 750-Da PEG AuNPs. However, PEGylated GNPs suffered from "accelerated blood clearance $(\mathrm{ABC})$ phenomenon" after repeated injection, and consequently, they fail to retain their sustained circulation (Zhao et al., 2017). Interestingly, inspired by the natural long circulation of red blood cells (RBCs), RBC-camouflaged gold AuNCs (RBCAuNCs) (Figure 6A) exhibited a superior circulation half-life than their biopolymer-stealth-coated counterparts (Figure 6B) and kept good colloidal stability in a mouse model. Moreover, mice treated with RBC-AuNCs achieved 100\% survival over a span of 45 days, whereas those receiving PVP-AuNCs or PBS exhibited loss of survival to varying extents (Figure 6C; Piao et al., 2014).

Although little to no dissolution and excretion of large gold nanoparticles $(\sim 10-200 \mathrm{~nm})$ in blood ensures long circulation time, gold's poor biodegradability induces its impaired physiological clearance in vivo. In order to improve this concern for clinical application, ultrasmall gold nanoparticles 


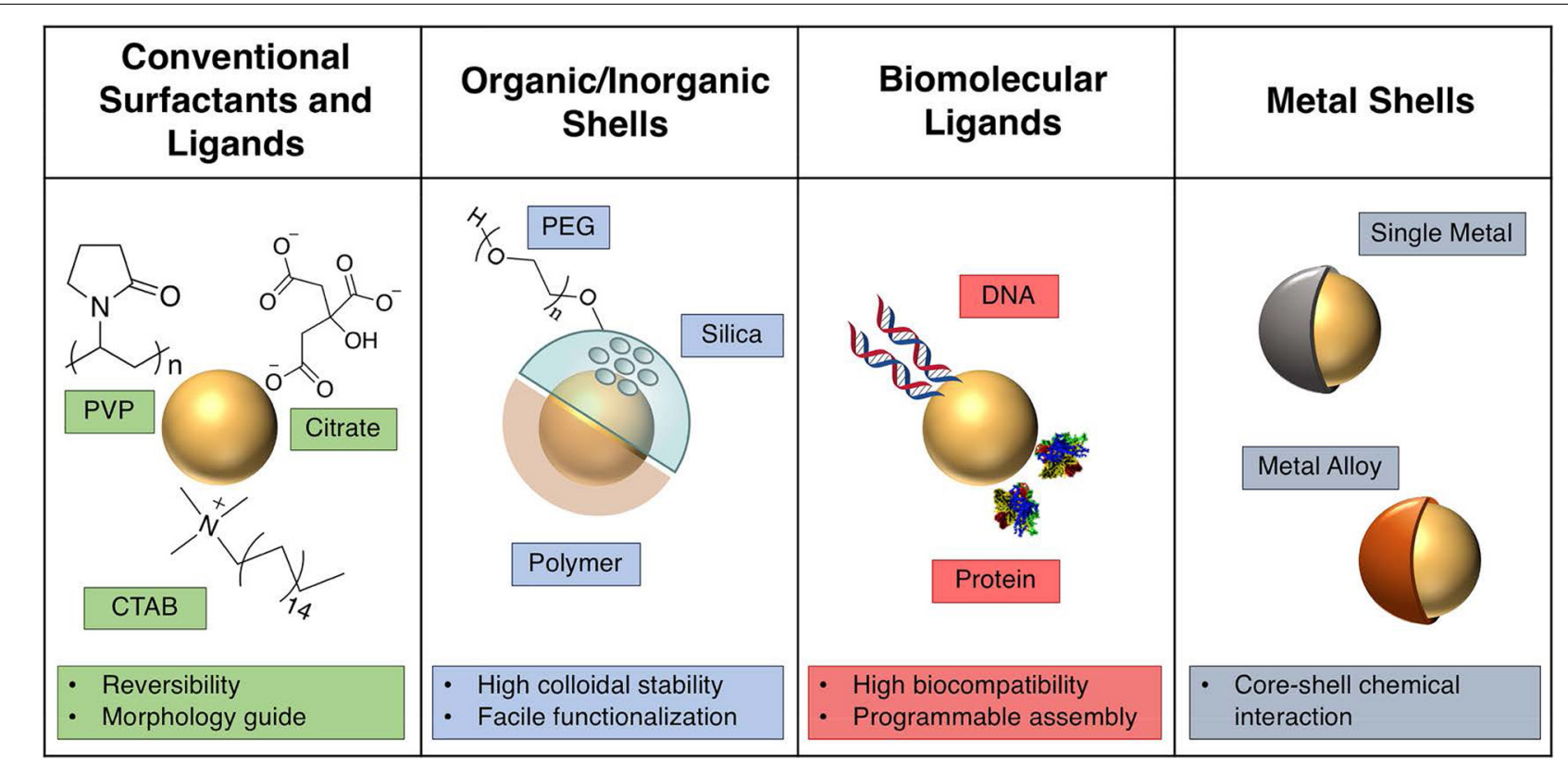

FIGURE 5 | Description of various strategies in colloidal plasmonic nanoparticle. Reprinted with permission from Kang et al. (2019), Copyright (2019) American Chemical Society.

A

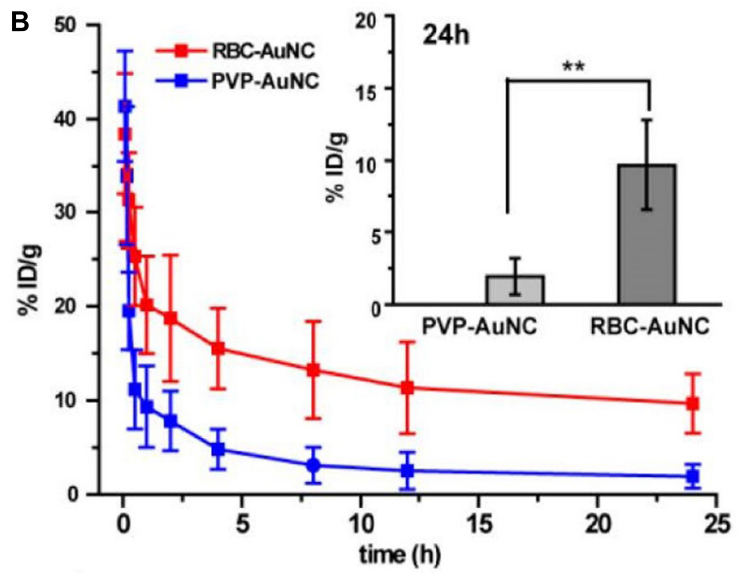

PVP-AuNC RBC-AuNC

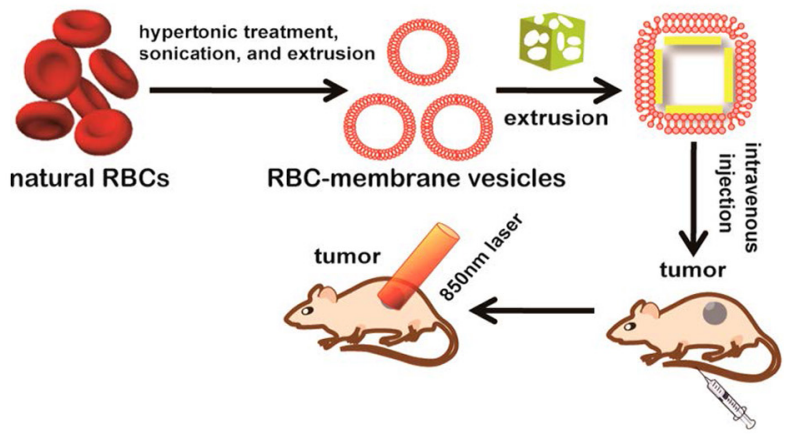

C



FIGURE 6 | (A) Schematic demonstrating the preparation of RBC-AuNCs.(B) The RBC-AuNCs retained longer in blood than the pristine PVP-AuNCs in mice. ${ }^{\star \star} P<0.01$. (C) The survival time of the RBC-AuNCs group was longer than that of PVP-AuNCs and PBS group. Reprinted with permission from Piao et al. (2014), Copyright (2014), American Chemical Society. 
are designed to be coated with the $\mathrm{pH}$-sensitive polymer and encapsulated within $\sim 150 \mathrm{~nm}$ of polymeric micelle. Micelles were inactive during long blood circulation and showed rapid release from the micelle in acidic buffers $(\mathrm{pH}$ 5.0) and in cultured macrophage cell. Mice receiving micelle treatment displayed progressive physiological clearance of GNPs, with the liver elimination $>85 \%$ over 3 months (Higbee-Dempsey et al., 2020).

\section{Passive and Active Targeting}

The tumor microenvironment plays a key role in determining passive targeting of GNPs (Figure 1). Tumor vessels defect a wellorganized branching hierarchy from large vessels into smaller vessels, and the structure of the vessel wall is abnormal, with an abnormally thick basement membrane, wide interendothelial junctions, a large number of fenestra, and the maximum pore diameters are several hundreds of nanometers (Golombek et al., 2018). Properly formed blood vessels in normal tissue does not allow nanoparticles to pass through because their size is relatively large compared with natural small molecules and growth factors. Therefore, the vascular permeability and hydraulic conductivity of tumors are significantly higher than normal tissues (Yhee et al., 2017). For this reason, after administered intravenously, GNPs easily extravasate through leaky vasculature and accumulate in the tumor. Besides, the lymphatic drainage in tumor tissue is highly impaired and does not drain the fluid efficiently contributing to nanoparticle entrapment and retention. This above phenomenon is often referred to as the enhanced permeability and retention (EPR) effect (Nel et al., 2017).

It is worth noting that inter-endothelial gaps do not account for the transport of nanoparticles into solid tumors, and up to $97 \%$ of nanoparticles extravasate into tumors through active trans-endothelial mechanisms (Sindhwani et al., 2020). The term "active targeting" often refers to specific bioconjugation (antibodies or small molecule) grafted to the nanomedicine surface that can specifically recognize tumor and bind to overexpressed receptors with a high affinity in the target region, then mediate nanomedicine-cell interactions, and subsequently enhance therapeutic efficiency (Byrne et al., 2008; Figure 1). These proteins or small biomolecules enable the favorable characteristics of gold nanoparticles to be linked with the biocompatibility, biodistribution, and physical properties (Li L. et al., 2019; Sharifi et al., 2019). Self-assembled monolayers (SAMs) have been applied in modifying the surface of GNPs. SAMs have the possibility to assemble on the surfaces of any shape or size, and they provide a reasonably strong and flexible method to precisely tailor the interfaces between gold nanoparticle structures and molecules in the surrounding environment (gold-sulfur bonds are stable to physiologically salt concentrations and relevant $\mathrm{pH}$ ) (Schon et al., 2003; Love et al., 2005).

\section{Biodistribution and Biocompatibility}

Biodistribution or biodurability is referred as the tendency of NPs to resist dissolution and biotransformation in media or physiologically relevant solutions (Bourquin et al., 2018), controlled by many factors including physicochemical properties (e.g., size and shape), doses administered, administration routes of GNPs, as well as tissue-/blood-dependent permeability. AuNPs do not universally target all cell types. For instance, the surface chemistry of the AuNPs influences the suborgan biodistributions. Positively charged AuNPs preferentially accumulated in the glomeruli of the kidneys, guarantying that they are rapidly cleared from circulation within $24 \mathrm{~h}$ of injection, faster than neutral and negatively charged NPs (Figures 7A,B). Neutral AuNPs had a greater extent of accumulation in the marginal zone with white pulp regions of the spleen and Kupffer cells of the liver, suggesting that these AuNPs extensively interact with the immune system, whereas positively and negatively charged AuNPs did not interact with the immune system as they broadly accumulated in the red pulp of the spleen (Elci et al., 2016). On the other hand, physicochemical parameters are key determinants in biodistribution in vivo, greatly affecting the kinetics of excretion and accumulation of AuNPs in filter organs (Figures 7C,D). Spherical and star-like AuNPs showed the same trend of accumulation except in the liver, and their ability to penetrate were higher than the rod-like AuNPs. In addition, star-like AuNPs were exclusively able to accumulate in the lungs (Figure 7E). Very interestingly, changes in the geometry did not influence poor accumulation in the kidneys of the GNPs themselves and the passage in the blood-brain barrier (Talamini et al., 2017).

The mononuclear phagocytic system MPS and renal system compete with the tumor for circulating NPs and eliminate the most of administered nanoparticles. The MPS is mainly composed of organs containing resident phagocytic cells, such as the liver and spleen. In the liver, Kupffer cells can recognize the opsonin adsorbed on the GNPs flowing through the liver sinusoidal capillaries and phagocytize them (Fischer et al., 2010). The macrophages in the spleen can potentially engulf circulating nanoparticles, but the specifics have not been fully investigated. The epithelium lining of the kidney contains filtration slits of 4$6 \mathrm{~nm}$ in width (Venkatachalam and Rennke, 1978), and GNPs smaller than this size may be filtered out of the blood and eventually excreted in the urine. Other major organs including the lymph nodes and the skin also have the ability to remove nanoparticles from the bloodstream.

Sufficient biocompatibility to ensure the innate functions of the normal cells are not disrupted or impaired, and is critically important for nanomaterial application in vivo. Gold nanoparticles are not biodegradable and would retain inside the body for long periods of time after administration. Despite tremendous potentials of clinical applications, the toxicity of AuNPs remains a controversial and important issue. Various researches consider that some factors such as surface medication, concentration, and physical dimensions (Pan et al., 2007) play an important part in affecting the cytotoxicity of the gold nanostructures. Surface functionalities are the major player in governing biocompatibility of GNPs, which are crucial for their successful implementation into the clinic. Studies indicate that some precursors such as CTAB, which is bound to the gold nanoparticles might be harmful to the cell, but the nanoparticles themselves are non-toxic (Connor et al., 2005; Li et al., 2018). Connor et al. (2005) found that 18-nm gold nanoparticles containing the CTAB displayed significant toxicity 
A

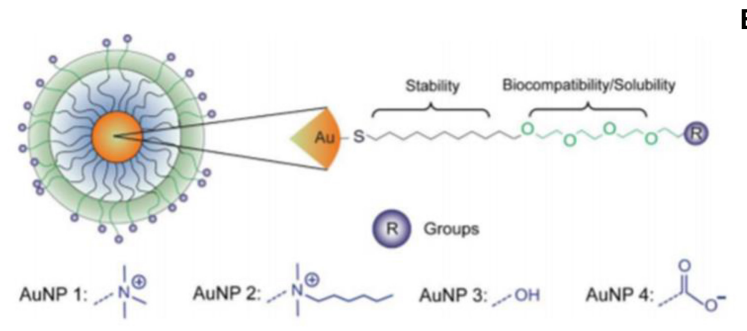

B



D

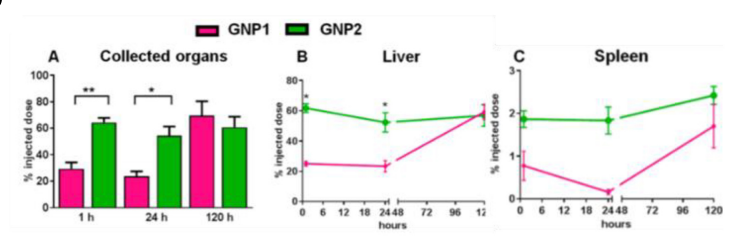

C

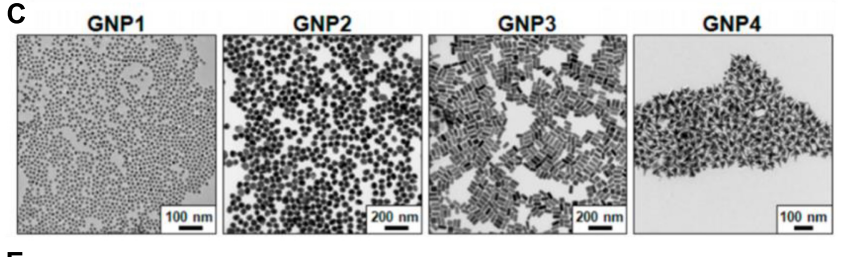

E

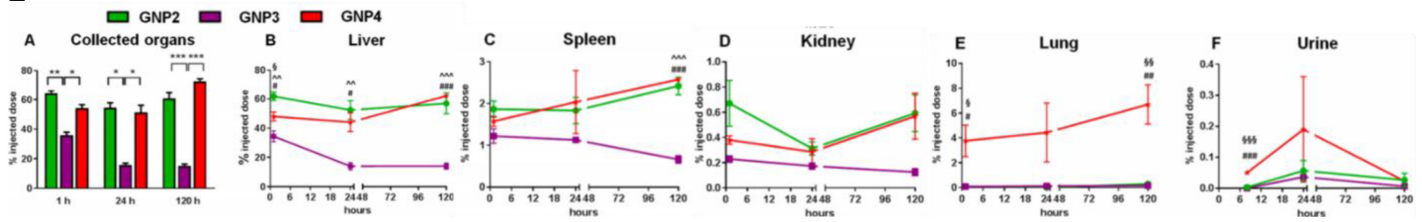

FIGURE 7 | (A) Structure of the different surface charge AuNPs. (B) Positively charged AuNPs (a,b,e,f) accumulated in glomeruli of the kidney tissues (c,d), while neutral ones $\mathbf{( g , h )}$ were in arteries of the kidney, and negatively charged AuNPs (i,h) were more homogeneously distributed. Reprinted with permission from Elci et al. (2016). Copyright (2016), American Chemical Society. (C) TEM micrographs of the AuNP library. (D) (A) Different sizes influence thekinetics of AuNPs. (B,C) The larger size penetrated faster in organs and remained stable a long time. (E) The biodistribution (A) of three shapes AuNPs with similar size, spherical, and star-like AuNPs had similar behavior in filter organs (B-D) and higher penetration than rod-like AuNPs. Star-like AuNPs showed a high tropism for the lungs (E) and higher levels in urine (F). ${ }^{\star} P<0.05,{ }^{\star \star} P \leq 0.005$, and ${ }^{\star \star \star} P \leq 0.0005\left({ }^{\wedge} \mathrm{GNP} 2\right.$ compared to GNP3, ${ }^{\S}$ GNP2 compared to GNP4, and \#GNP3 compared to GNP4). Reprinted with permission from Talamini et al. (2017), Copyright (2017), American Chemical Society.

similar to the cytotoxicity of CTAB alone. They centrifuged and washed the CTAB-modified nanoparticles with deionized water three times to wipe out free $\mathrm{CTAB}$ in order to find whether the CTAB-modified nanoparticles or unbound CTAB induced the observed cytotoxicity. The results revealed, under the conditions examined, that washed CTAB-modified nanoparticles were detected to be not toxic, which suggested that the coating molecules' cytotoxicity was attributed to the toxicity of the gold nanoparticles (Connor et al., 2005). Fraga et al. (2014) have found that two different modifications with the same hydrodynamic size and surface charge, capped on the AuNPs, had a very similar distribution pattern at each time-point and mainly accumulated in the hepatic and spleen tissues. Through examining the electron-dense deposits and major clinical changes, they proposed that the surface chemistry seems to have more effect on the toxicity rather than on the biodistribution of short term and long term in vivo (Fraga et al., 2014). Zhang et al. (2011) found that the 10- and 60-nm PEG-coated AuNPs induced a significant increase in aspartate transaminase and alanine transaminase levels; meanwhile, the decrease in creatinine was observed in mice treated with the $60-\mathrm{nm}$ particles, which indicated the damage to the liver and kidneys. This article also noted that the preference distribution of different size particles may affect the immune system, but even these particles broke down in vivo, and they did not cause appreciable toxicity (Zhang et al., 2011). Conversely, Kharazian et al. (2018) used molecular dynamic simulation (MD) to study the interaction of GNPs with fibrinogen (Fg), and their results revealed that the bare gold surface has a critical role in inducing Fg conformational changes regardless of the type or presence of a surface coating. They also reinforced the MD results in ultrasmall gold ( $\sim 10 \mathrm{~nm}$ or smaller). Although the surface coatings either partially degraded in biological media or contained gaps due to disorganization integrity of the surface coverage, NPs still had the capacity to induce the unfolding of Fg. These results potentiate concerns about the unforeseen immunoreaction of GNPs in vivo (Kharazian et al., 2018).

Besides, intracellular gold nanorods (GNRs) are recently reported to have perturbed the ionic microenvironment within the nucleus and altered gene expression in human cells. Despite GNRs were localized in the cytoplasmic vesicles with no genomic DNA interaction, intracellular localization could lead to the separation of gold species from the GNR surface into the nucleus, resulting in structural alterations of genomic DNA (Ho et al., 2018). The cytotoxicity of AuNRs in vascular smooth muscle cells (VSMCs) was strongly correlated with the binding forces and the binding probability between the AuNRs and the cell membrane. Poly(diallyldimethyl ammonium 
chloride-coated AuNRs) (PDDACAuNRs), having a relatively large size binding force and large binding probability, were non-toxic in cancer cells in previous reports, whereas it displayed extreme toxicity to VSMCs and was detrimental to VSMC contractile function. When the rational design of nanoparticles for medical applications are considered, it is necessary to assess the cytotoxicity of gold nanoparticles on cells, as the cytotoxicity of AuNRs is cell specific (Sun et al., 2018).

\section{DETECTION FOR CANCER}

\section{Gold Nanoparticles in Biomarker Detection}

Due to the remarkable plasmonic properties and large surface area to volume ratio of gold nanoparticles, they have been widely investigated for their potential applications in biomarker detection. Biomarkers may be molecules or something overexpressed in blood and serum or at the region of cancer that facilitate diagnosis. Detection of some biomarkers occurring in ultralow levels is challenging and require ultrasensitive detection strategies (Viswambari Devi et al., 2015).

Nanozymes have shown a broad range of applications in in vitro detection (Zhu et al., 2019). GNPs as a nanozyme material, compared with platinum and gold-platinum bimetallic hybrid nanoclusters, possesses the highest catalytic activity and low coefficient of production variation $(8.5 \%)$, and can be designed as a scalable excellent diagnostic platform. The smallersized nanomaterials exposing higher surface-to-volume ratio and more active sites have a better catalytic activity (Wu et al., 2019). Ultrasmall ( $<2 \mathrm{~nm}$ ) AuNCs mastering intrinsic catalytic activity and efficient renal clearance properties, with the presence of surface peptide modification, are able to monitor a diverse range of diseases in the urine (Zhang et al., 2015). After exposure to complex physiological environments, such as patient serum and urine, AuNCs still retained a high stability and effectively evaded non-specific protein adsorption. Neutravidin (NAV), which have low non-specific binding and high affinity for biotin properties (Jain et al., 2017), coupled to the catalytic AuNCs, forming MMP-responsive nanosensors, specifically cleaved MMP or the serine protease thrombin. In tumor-bearing mice, AuNC-NAV complexes could be actively disassembled by elevated MMP levels at the site of the disease, and then proteolytically liberated AuNCs, by virtue of their small size, were efficiently filtered into the urine to be analyzed by TMB catalytic activity assay and ICPMS (Figures 8A,B). This system can be developed to diagnose a range of diseases in microenvironments (Loynachan et al., 2019).

Moreover, gold nanoparticles can detect genetic mutations in tumor. The single NP-sensing (sNPS) technology is a promising approach to achieve non-invasive assays for elucidating subtle variations in genes (Li L. et al., 2019). The sensing scale of LSPR in gold nanoparticles can be reduced to a single NP, which owns a high-throughput signal-to-noise ratio $(\mathrm{S} / \mathrm{N})$. The refractive index of single NP relies on the structure and localized sensing volume/area of the NP. Gold-bridged NPs as a signal source under low-energy white light showed twofold higher sensitivity than gold nanorods. Furthermore, the ionic environment of the DNA interface can alter Au-bridged NPs. When mutation sequence non-specifically bind to point mutations, LSPR signals change and different point mutations own variable reactions (Figure 8C). Through the sNPS system and the typical atlas, this product sensors can effectively and directly identify subtle distortions in biomolecular binding and genetic alterations (Ma et al., 2019).

Gold nanoparticles can act as SERS hotspots to detect cancer (Wang Z. et al., 2018). Volatile organic compounds (VOCs), serving as biomarkers of the non-invasive recognition of lung malignancies, are always difficult to detect for the reason that high velocity of VOCs makes a low absorptivity on solid substrates and weak Raman scattering. The ZIF-8 layer, providing a porous character, was coated onto a self-assembly of gold superparticles (GSPs) to form a GSPs@ZIF-8 SERS substrate, could affect the traveled fashion and process of gaseous molecules, and induce high-intensity electromagnetic fields. Through Schiff base reactions, the pregrafted amino group of 4-ATP in GSPs@ZIF-8 sensitively captured gaseous aldehydes (released at tumor-specific tissue) at the ppb (parts per billion) level, providing a promising opportunity for in vitro diagnoses of early stage lung cancer (Qiao et al., 2018).

\section{Imaging \\ Photoacoustic Imaging}

Photoacoustic (PA) imaging, is a non-invasive imaging modality, obtaining real-time information based on converting absorbed short-pulsed laser energy into ultrasound through the thermoelastic effect. These ultrasound waves can be detected by ultrasound transducers at the tissue surface that converts the mechanical acoustic waves to electric signals and after duly processing to form an image representing the absorbed optical energy (Fu et al., 2019). Photoacoustic imaging has better tissue contrast related to the optical properties of different tissues, without the limitations in the mechanical properties of biological tissues existing in ultrasound imaging. AuNPs possess a high molar extinction coefficient, which can maximize the amount of light absorbed, optimal tissue penetration to avoid the strong absorption of intrinsic chromophores, and are not susceptible to photobleaching (Qiu et al., 2020). PAI choosing AuNPs as exogenous contrast agents can indicate the presence of various tumors by monitoring the passive or active accumulation of receptors which are functionalized on the AuNPs in the tumor, or map sentinel lymph nodes to trace the metastasis of cancer cells (Dumani et al., 2019), and provide a specific target in tracking intravascular PA (IVPA) imaging of macrophages in atherosclerotic plaques (Hui et al., 2017). PA imaging can track stem cells based on gold nanoparticles as a contrast agent. GNPs have good loading into stem cells and are not appreciably exocytosed (Figure 9A). The contrast agent system consists of inert gold nanorods bound with ROS-sensitive near-infrared dye IR775c (stem cells produce ROS to degrade the cell). This nanoplatform can track cell viability in real time with high spatial and temporal resolution, and assess the contribution and efficacy of cell therapies (Dhada et al., 2019). Besides, AuNPs encapsulated within the lipid shell of microbubbles 
A
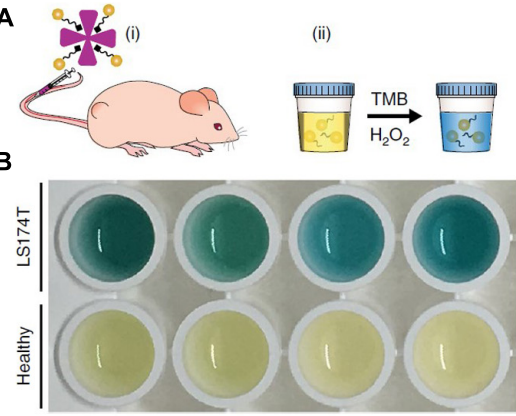

C

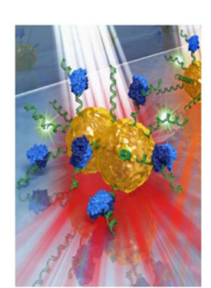

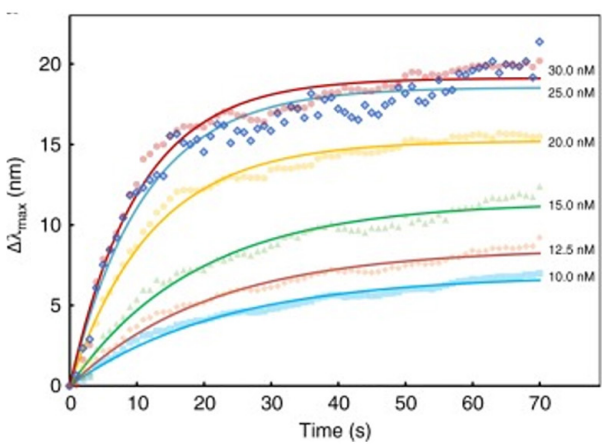

FIGURE 8 | (A) Schematic of the renal clearance assay, including two steps (i and ii). (B) Colorimetric assay of urine from tumor and healthy-bearing mice injected with AuNC-P220-Nav. The blue color urine is belonging to samples from tumor-bearing mice. Reprinted with permission from Loynachan et al. (2019), Copyright (2019), Springer Nature. (C) Illustrations of single NP sensing (sNPS) models and real-time monitoring of MutS binding to each point mutation and a homoduplex DNA, non-specific sequence binding of MutS to point mutations change LSPR signals. Error bars represent mean \pm SEM. Reprinted with permission from Ma et al. (2019).

A

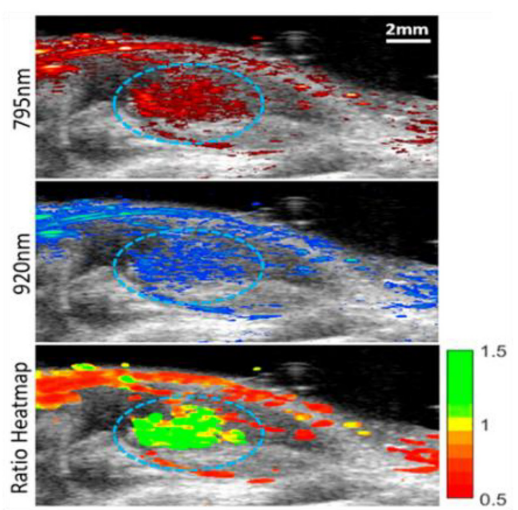

C


B

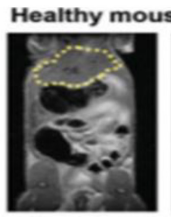

o h

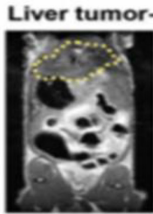

o h

$\overline{\text { Pre-injection }}$

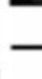

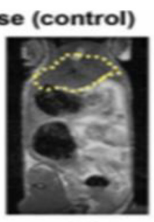

$1 \mathrm{~h}$

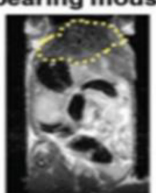

$1 \mathrm{~h}$

D
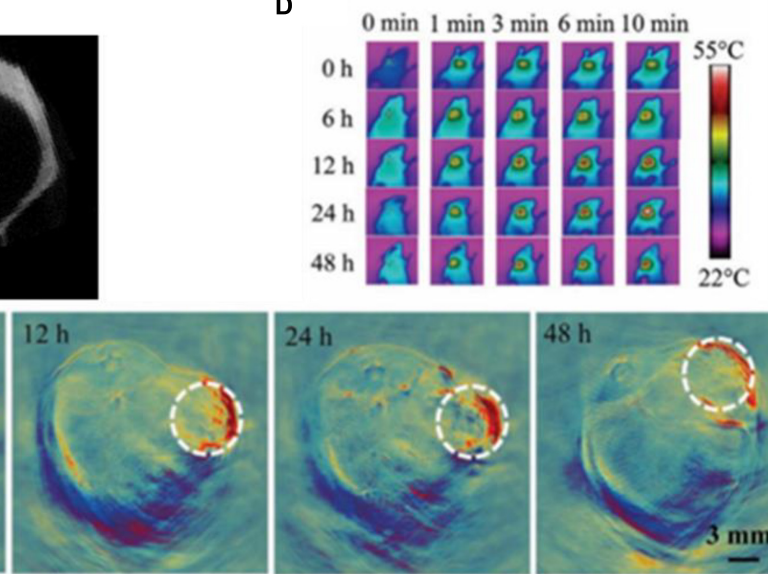

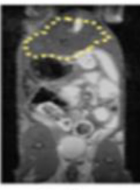

$3 \mathrm{~h}$

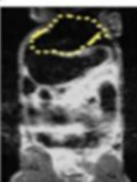

$3 \mathbf{h}$

$6 \mathrm{~h}$

FIGURE 9 | (A) The US/PA image of labeled stem cells (circled in blue) in vivo. Reprinted with permission from Dhada et al. (2019), Copyright (2019), American Chemical Society. (B) MRI imaging using SPAuNC (aff+) as a contrast agent from the mice bearing a liver tumor and healthy mice; the tumor-grafted liver was clearly differentiated from the normal liver of healthy mice. Reprinted with permission from Kwon et al. (2017), Copyright (2017), John Wiley and Sons. (C) In vivo micro-CT images of AuNP-PLL-RITC-labeled hMSCs. Reprinted with permission from Kim et al. (2017), Copyright (2017) John Wiley and Sons. (D) Photothermal and (E) photoacoustic imaging of Capan-1 tumor-bearing nude mice injected with rGADA. Reprinted (adapted) with permission from Jia X. et al. (2020), Copyright (2020) John Wiley and Sons. 
(MBs) can act as a US-responsive PA imaging probe for in vivo "background-free" PA imaging. AuNPs separated by the lipid shell of MBs showed relatively low NIR PA signals. Whereas Au@lip MBs were exposed to US pulses, they would burst into nanoscale aggregates of Au@lip NPs and exhibit significantly enhanced NIR photoacoustic signals due to their red-shifted surface plasmon resonance absorbance. The tissue background PA signals could be realized by subtracting the PA image captured pre-US burst from that after US burst, enabling background-free PA imaging with high sensitivity. As a proof of concept, Au@lip MBs as a multimodal probe for US-responsive background-free PA imaging is a particularly promising strategy for detection of changes in tumor blood perfusion after drug treatment (Meng et al., 2019).

\section{Magnetic Resonance Imaging}

Magnetic resonance imaging (MRI), as the widely used diagnostic modalities in the clinic, is non-ionizing, non-invasive, and radiation free. Under an external magnetic field, magnetic nuclei align to allow resonance through a radiofrequency pulse (Zhou et al., 2019). The resolution of MRI for disease diagnosis can be affected by using MRI contrast agents that improve the contrast by shortening the longitudinal $\left(\mathrm{T}_{1}\right)$ or transverse $\left(\mathrm{T}_{2}\right)$ relaxation times of protons (Jeon et al., 2020). Although superparamagnetic iron oxide nanoparticles (SPION) were used to be applied as contrast agents of $\mathrm{T}_{2}$-weighted MRI, clinics no longer allow SPIONs in vivo because of their ROS toxicity causing severe cellular damage and inflammatory responses (Kwon et al., 2017). The GNPs with anisotropic geometries and shapes elevate local field inhomogeneity under an external magnetic field (Yang et al., 2019). The glutathione (GSH)-responsive $\mathrm{Au}$ nanowreath (AuNW) effectively guided magnetic imaging (Figure 9B). Exceedingly small magnetic iron oxide nanoparticles (ESMIONs) decorated on AuNWs make this nanostructure not merely effectively quenched with the $\mathrm{T}_{1}$-weighted MRI due to the strong $\mathrm{T}_{2}$ decaying effect but also responded to glutathione (GSH) and had the $T_{1}$ imaging function "turning on." After intravenous injection, the relatively high GSH concentration in tumor microenvironment induced the switch of $\mathrm{T}_{1}$ signal of magnetic AuNWs from an initially "OFF" state to "ON" state. The larger assemblies of ES-MIONs enhanced their tumor accumulation compared with ES-MIONs alone, providing higher MRI contrast imaging (Kwon et al., 2017).

\section{Computed Tomography}

Another commonly employed non-invasive clinical imaging technique is computed tomography (CT), which relies on the $\mathrm{X}$-ray attenuation by the tissues (Lee et al., 2013). AuNPs are promising CT contrast agent materials because gold has a high atomic number and density, thus, possessing an intrinsic high X-ray absorption coefficient (Sabale et al., 2017). Citratestabilized GNPs coated with poly-L-lysine (PLL) and rhodamine $\mathrm{B}$ isothiocyanate (RITC) enable CT cell tracking in vitro and in vivo (Figure 9C; Kim et al., 2017) and may provide applications in CT image-guided interventions utilized in the injection of cellular therapeutics.

\section{Photothermal Imaging}

The photothermal effect can be detected by a thermal imaging system to form photothermal imaging (PTI). PTI requires a heating beam and a probe beam to detect refractive index variations induced by their increase in the local environment. Its high sensitivity of detection is at the single-particle level in a dark background. Gold nanoparticles are ideal probes for PTI due to their biocompatibility, fast relaxation times, and large absorption sections around plasmon resonance (Willets et al., 2017). Wang et al. introduced rGO@AuNSDODAB/DOPE-FA (rGADA) for photoacoustic/photothermal dual-modal imaging-guided photothermal and gene synergistic therapy of pancreatic cancer. From photoacoustic imaging in Capan-1 tumor-bearing mice, the facilitated accumulation of the rGADA in tumors displayed considerably distinct photoacoustic signals (Figure 9E). At the same time, the PTI showed that the temperature of tumor increased promptly to above $55^{\circ} \mathrm{C}$ (Figure 9D), which was relatively high enough for the ablation of the tumor. These results suggested that the combination of reduced graphene oxide (GO) and AuNS improved the dispersity and photothermal efficiency (Jia X. et al., 2020).

\section{GOLD NANOPARTICLES IN THERAPY}

\section{Drug Delivery}

In cancer chemotherapy, anticancer drug resistance can be intrinsic (primary) or acquired after repeated cycles of chemotherapy (secondary). Chemotherapeutic drugs fail to activate or alter drug-appointed targets and enhanced drug efflux from the drug accumulation regions, are the established mechanisms that markedly lower anticancer efficiencies in tumors (Lepeltier et al., 2020). In the current researches of cancer medicine, significant efforts have been devoted to explore smart nanoparticles for an effective targeted drug delivery system (TDDS) (Luther et al., 2020). In designing nanomedicines, there are several points to be considered, such as preferential concentration in the tumor tissue, efficient internalization into cancer cells, and accurate subcellular location of drugs at their site of actions. GNPs can be utilized as complex systems, including nanocarrier-mediated combination therapies maximizing the therapeutic efficacy and NIR light-induced drug-responsive release to overcome mechanisms of drug resistance (Sharifi et al., 2019). Although tumor microenvironment heterogeneities cause many obstacles to successful GNP delivery such as the clearance of immune cells and reduced permeation, researchers designed the nanomaterial drug delivery systems based on the characteristics of the tumor microenvironment to enhance therapeutic efficacy and accuracy.

Due to hypoxia in the tumor microenvironment and glycolysis as the main strategy for glucose metabolism, the cancer cells are trapped in acidic extracellular micromilieu (McDonald et al., 2019). An effective anticancer $\mathrm{pH}$-sensitive nanosystem should have the ability to store and stabilize the drug at physiological $\mathrm{pH}$, rapidly release the payloads at the trigger point $\mathrm{pH}$, and ensure a therapeutic dose of drug concentration at the 
intracellular level. There are usually three strategies for $\mathrm{pH}$ responsive drug release in the tumor microenvironment: the first approach is to utilize chemical groups wherein the changes in physical or chemical properties depend on $\mathrm{pH}$ and accept or donate protons; the second approach is to apply acidlabile chemical covalent bonds between drug molecules and the surfaces of nanocarriers, or to construct new nanocarriers (Liu et al., 2014); and the third method is the $\mathrm{pH}$-responsive "PEG detachment"(Kanamala et al., 2016).

The acidic conditions of the tumor microenvironment (TME) inspire tumor immunotherapeutic efficacy. Jibin Song's group developed dual $\mathrm{pH} / \mathrm{GSH}$-responsive nanogapped gold nanoparticle vesicles carrying an immune inhibitor (BLZ945) and an anticancer polymeric prodrug for synergistic concurrent chemo-immunotherapy. The vesicles were fabricated by self-assembly of amphiphilic GNPs grafted with hydrophilic PEG and dual $\mathrm{pH}$ and redox-response copolymerized prodrug PSN38VP [poly (SN38-co-4-vinylpyridine)], which contained the reduction-responsive disulfide $(-\mathrm{S}-\mathrm{S}-)$ bond and $\mathrm{pH}$ responsive 4-vinylpridine (4-VP). The theranostic nanoplatform also can guide cargo release by showing photoacoustic (PA) intense PA signal in the NIR-II window. The responsive AuNNP@SN38/BLZ-945 released nearly 90\% of BLZ-945 at pH 6.5 , whereas negligible leakage of BLZ-945 was detected at pH 7.4, suggesting that AuNNP@SN38/BLZ-945 could selectively release BLZ-945 at tumor $\mathrm{pH}$ and maintain stability in neutral $\mathrm{pH}$. Following dissociation of the $\mathrm{pH}$-responsive nanovesicles, the PA signal intensity decreased due to attenuation of the plasmonic coupling between GNPs and the "blue-shift" of absorbance. The characteristic of GSH-responsive released SN38 in the reductive microenvironment exerted cytotoxic effects. Therefore, GNPs as nanocarrier and imaging agents, combined with $\mathrm{pH} / \mathrm{GSH}$ responsive, have a superior nanostructure in inhibiting the growth of tumor (Zhu et al., 2020).

In subcellular acidification compartments, a nanoparticledirected drug can be delivered to the endosomes and lysosomes for pH-responsive intracellular release (Liu et al., 2014). The pH-responsive system can utilize oligodeoxynucleotides (ODNs) packed on the nanoparticles to modulate the hybridization stability controlling the assembly and disassembly of nanoparticles in neutral or acidic $\mathrm{pH}$, and its duplex structure can be utilized to load intercalative drugs. Based on the features mentioned above, Won Jong Kim and his workers designed the cytosine (C)-rich i-motif sequence to link sAuNPs for clustering into size-tunable large clusters (cAuNPs), and i-motif sequence was added into iBO-sAuNPs at neutral $\mathrm{pH}$, causing the formation of cluster. In an acidic $\mathrm{pH}$, the cytosine (C)-rich i-motif formed a unique tetrameric structure by partial hybridization of $\mathrm{C}$ and protonated C. Following cellular uptake, the i-motif and iBO-sAuNPs dissociated at acidic endosomal pH. Taken together, this dynamic DNA nanocluster provided higher tumor specificity and efficient drug release (Kim et al., 2014).

Hamishehkar's group developed a new type of smart core/shell of gold NPs decorated by a pH-responsive copolymer of thiolated polyethylene glycol-b-poly to target and trigger the delivery of MTX. Amino and thiol side groups in the backbone of a copolymer consisting of a polymeric shell were used to couple a polymer and a load of MTX to the surface of gold NPs. Moreover, due to the folate-mediated endocytosis behavior of MTX, this developed NPs were valued for their potential in efficient cell uptake. Under the acidic condition of a simulated cancer tissue $\left(\mathrm{pH} 5.3,40^{\circ} \mathrm{C}\right)$, MTX was released three times faster than the physiological state $\left(\mathrm{pH} 7.4,37^{\circ} \mathrm{C}\right)$, and $78 \%$ of the MTX was released from the nanocarriers. Nevertheless, only $26 \%$ of the loaded MTX was released in physiological conditions $\left(\mathrm{pH} 7.4,37^{\circ} \mathrm{C}\right)$ after $48 \mathrm{~h}$. These results suggest that polymers with the ionizable functional groups grafted on the GNPs could be promising $\mathrm{pH}$-responsive systems (Ghorbani and Hamishehkar, 2017).

\section{Photodynamic Therapy}

The hypoxic tumor microenvironment limits the ROS generation of PDT due to oxygen deficiency (Zhou et al., 2016). Increased cellular metabolism, mitochondrial dysfunction, and oncogene activity promote the excessive accumulation of ROS in cancer cells. The major source of endogenous ROS is continually produced in the mitochondria. Electron leakage from the electron transport chain in the mitochondria, primarily in the reaction process mediated by coenzyme $\mathrm{Q}(\mathrm{CoQ})$, ubiquinone, and its complexes, may react with molecular oxygen to release mitochondrial superoxides, which can subsequently be converted to other ROS through chemical reactions. Overproduction of ROS can oxide cellular components, destruct the mitochondria, and disturb normal metabolism leading to cytotoxicity. Unfortunately, although the ROS level in TME is relatively high compared with that of the normal tissues, the amount of endogenous ROS is insufficient to stimulate rapid release of large quantities of monomeric drugs (Zhang L. et al., 2018). The ROS-responsive systems combining the PDT would serve as a powerful exemplary approach to overcome the aforementioned drawbacks. The hyaluronidase-responsive size-reducible biomimetic nanoparticles (mCAuNCs@HA) was a synergetic combination therapy for chemotherapy, PDT, and immunotherapy. In this study, the photosensitizer pheophorbide A (PheoA) and ROS-responsive prodrug PXTK were coloaded to facilitate drug release. The synergetic effect of PDT-induced immunogenic apoptosis and PD-L1 blockade induced immunosuppressive boosted host immune system. The combination therapy provided extraordinary tumor inhibition and antitumor immune response (Yu et al., 2019).

Gold nanoparticles can act as a vehicle to carry a high payload of photosensitizers, forming the GNR-photosensitizer complex. The complex using the target tissue receptors or antigens modified on the surface of GNR passively localize on the diseased site. With the PS released from the GNR, the NIR fluorescence imaging detect the tumor with a high signal-to-background ratio, and the tumor can be selectively destroyed in a non-invasive manner with minimizing phototoxic damage of the surrounding normal tissues in photodynamic theranostic (Gamaleia and Shton, 2015). Besides, the presence of gold nanoparticles in the complex improved the quantum yield of singlet oxygen production significantly. Instead of acting as a transport carrier activating PSs in the vicinity, gold nanoparticles without the presence of any organic photosensitizers, can directly sensitize 
the generation of singlet oxygen through irradiation with light. Recently, AuNCs, composed of several to hundreds of gold atoms and generally $<2 \mathrm{~nm}$ in diameter (Higaki et al., 2019; Zhou et al., 2019), have multiple peaks in their ultraviolet-visible absorption spectra and discrete electronic energy levels, which sharply distinguish them from AuNPs (Gao et al., 2019). AuNCs emerge as a promising ideal PS candidate for PDT. For the reasons that AuNCs are chemically pure and have eco-friendly synthesis (Poderys et al., 2020), they exhibit excellent biocompatibility and low toxicity, long tumor retention and rapid normal tissue clearance, and the microsecond ( $\mu s)$ range fluorescence lifetime is conducive to the generation of excess ROS. Dihydrolipoic acidcoated gold nanocluster (AuNC@DHLA) possessed superior two-photon optical properties and strong ROS generation when stimulated by an external laser, and showed highly efficient antitumor effects in a hepatocellular carcinoma xenograft model with negligible toxicity (Han et al., 2020).

\section{Photothermal Therapy}

Despite advances in nanoparticle design of heating trials, most photothermal studies failed to take into account the effect of the tumor environment on light-to-heat conversion efficiency. Claire Wilhelm and his workers systematically studied the lightto-heat conversion efficiency of gold nanostars from aqueous dispersion to cancer cells cultured in vitro and injected to solid tumors in vivo. Their results elucidated that the heating efficiency of gold nanostars in aqueous dispersion was governed by particle size and laser wavelength, while the heat generation of nanostars internalized by cancer cells in vitro still appeared very efficient, but was size and laser independent. Meanwhile, the heat generation of nanostars intratumorally injected in vivo evolved with time in cellular internalization. Together, the common recorded photothermal conversion efficiencies in aqueous dispersion cannot predict the values acquired from either isolated cells or living tumor tissues, and the most important design features of gold nanostars for thermotherapy were their size and coating affecting optimal biodistribution in vivo rather than their plasmonic peak measured in aqueous dispersion (Espinosa et al., 2016).

\section{CONCLUSION AND OUTLOOK}

With the rapid development of nanomedicine, various organic or inorganic nanoparticles have been investigated, among which gold nanoparticles have attracted increasing attention due to its feasible functionalized surface, excellent tumor specificity, high drug-loading capacity, and biocompatibility. Multifunctional designs of gold nanoparticles enabling the in situ imaging such as computed tomography (CT) and MRI, or chemotherapy drug delivery and the anti-cancer effects of PDT and PTT, have been constructed. In this review, we discussed the optical and plasmon properties of gold nanoparticles, then elucidated their physical principle in detail. The physicochemical properties of GNPs support them to provide unique and sensitive nanoplatforms for imaging, diagnostic, and therapy. Of these GNP characteristics, localized surface plasmon resonance as the essential and powerful optical property is used through surface-enhanced processes including SERS, surface-enhanced fluorescence, and other plasmonic performances (Mejia-Salazar and Oliveira, 2018).

We briefly summarized the current applications of GNPs and introduced the collected researches of GNPs on theranostic systems in recent years, particularly emphasizing on these functional GNPs applications in TME. Before entering the tumor region, the GNPs experience the process of blood circulation and biodistribution. We demonstrated the series of biological behaviors in vivo and discuss the nanotoxicity of GNPs. Tumor environment is an indispensable field for anti-cancer nanotechnology researches, breaking away from which to do cancer treatment studies is meaningless. GNPs have been engineered to response or profit from particular aspects of the TME to acquire selective targeting of the tumor region, or an environment-responsive therapeutic cargo, and precisely accumulate at the appointed regions. However, such high-performance nanoplatforms, applied in the complex tumor microenvironment, remain intractably challenging. For example, except the well-known hypoxic microenvironment, the tumor microenvironment is also characterized by hypertonic characteristics. The elevated intertumoral penetrating pressure in the extracellular matrix (ECM) surrounding cancer cells can dramatically affect plasmonic properties of the GNPs that bring the quite variant optimal therapeutic properties between in model media and in vivo (Espinosa et al., 2016). Nevertheless, few researches focus on the hypertonic tumor media in the solid tumors. Additionally, the dense extracellular matrix (glycoproteins, proteoglycans, hyaluronic acid, etc.) and fibrotic tissue, which varies with tumor types, always hamper nanoparticles' access to targeted tumoral regions and limit their further penetration (Danhier, 2016). Moreover, GNPbased nanomedicines are still in preclinical stage with only few translations from the laboratory studies to clinical trials. More works are required to simulate the actual scenes of occurrence in tumor and elucidate the mechanisms associated with its applications in medicine. Overall, it is essential to explore the possibilities of gold nanoparticles in TME applications and make more theoretical breakthroughs in order to promote the process of nanotechnology applied in more clinical practice.

\section{AUTHOR CONTRIBUTIONS}

QG: writing-concept. JG, ZZ, and HZ: original draft preparation and discussion. JZ: artwork. QG and DW: review and editing. All authors: contributed to the article and approved the submitted version. 


\section{REFERENCES}

Agrawal, A., Cho, S. H., Zandi, O., Ghosh, S., Johns, R. W., and Milliron, D. J. (2018). Localized surface plasmon resonance in semiconductor nanocrystals. Chem. Rev. 118, 3121-3207. doi: 10.1021/acs.chemrev.7b 00613

Alkilany, A. M., Thompson, L. B., Boulos, S. P., Sisco, P. N., and Murphy, C. J. (2012). Gold nanorods: their potential for photothermal therapeutics and drug delivery, tempered by the complexity of their biological interactions. Adv. Drug Deliv. Rev. 64, 190-199. doi: 10.1016/j.addr.2011. 03.005

Amendola, V. (2019). Correlation of surface-enhanced Raman scattering (SERS) with the surface density of gold nanoparticles: evaluation of the critical number of SERS tags for a detectable signal. Beilstein J. Nanotechnol. 10, 1016-1023. doi: 10.3762/bjnano.10.102

Bhalla, N., Jain, A., Lee, Y., Shen, A. Q., and Lee, D. (2019). Dewetting metal nanofilms-effect of substrate on refractive index sensitivity of nanoplasmonic gold. Nanomaterials (Basel) 9:1530. doi: 10.3390/nano9111530

Bourquin, J., Milosevic, A., Hauser, D., Lehner, R., Blank, F., Petri-Fink, A., and Rothen-Rutishauser, B. (2018). Biodistribution, clearance, and long-term fate of clinically relevant nanomaterials. Adv. Mater. 30:e1704307. doi: 10.1002/adma. 201704307

Byrne, J. D., Betancourt, T., and Brannon-Peppas, L. (2008). Active targeting schemes for nanoparticle systems in cancer therapeutics. Adv. Drug Deliv. Rev. 60, 1615-1626. doi: 10.1016/j.addr.2008.08.005

Chan, C. H., Poignant, F., Beuve, M., Dumont, E., and Loffreda, D. (2020). Effect of the ligand binding strength on the morphology of functionalized gold nanoparticles. J. Phys. Chem. Lett. 11, 2717-2723. doi: 10.1021/acs.jpclett. 0c00300

Charbgoo, F., Nejabat, M., Abnous, K., Soltani, F., Taghdisi, S. M., Alibolandi, M., et al. (2018). Gold nanoparticle should understand protein corona for being a clinical nanomaterial. J. Control Release 272, 39-53. doi: 10.1016/j.jconrel.2018. 01.002

Chatterjee, D. K., Fong, L. S., and Zhang, Y. (2008). Nanoparticles in photodynamic therapy: an emerging paradigm. Adv. Drug Deliv. Rev. 60, 1627-1637. doi: 10.1016/j.addr.2008.08.003

Chen, H., Shao, L., Li, Q., and Wang, J. (2013). Gold nanorods and their plasmonic properties. Chem. Soc. Rev. 42, 2679-2724. doi: 10.1039/c2cs $35367 \mathrm{a}$

Chen, W., Zhang, S., Yu, Y., Zhang, H., and He, Q. (2016). Structural-engineering rationales of gold nanoparticles for cancer theranostics. Adv. Mater. 28, 85678585. doi: 10.1002/adma.201602080

Chen, Y. S., Zhao, Y., Yoon, S. J., Gambhir, S. S., and Emelianov, S. (2019). Miniature gold nanorods for photoacoustic molecular imaging in the second near-infrared optical window. Nat. Nanotechnol. 14, 465-472. doi: 10.1038/ s41565-019-0392-3

Chen, Y. Y., Syed, A. M., MacMillan, P., Rocheleau, J. V., and Chan, W. C. W. (2020). Flow rate affects nanoparticle uptake into endothelial cells. Adv. Mater. 32:e1906274. doi: 10.1002/adma.201906274

Cheng, L., Wang, C., Feng, L., Yang, K., and Liu, Z. (2014). Functional nanomaterials for phototherapies of cancer. Chem. Rev. 114, 10869-10939. doi: $10.1021 / \mathrm{cr} 400532 \mathrm{z}$

Connor, E. E., Mwamuka, J., Gole, A., Murphy, C. J., and Wyatt, M. D. (2005). Gold nanoparticles are taken up by human cells but do not cause acute cytotoxicity. Small 1, 325-327. doi: 10.1002/smll.200400093

Danhier, F. (2016). To exploit the tumor microenvironment: Since the EPR effect fails in the clinic, what is the future of nanomedicine? J. Control Release 244, 108-121. doi: 10.1016/j.jconrel.2016.11.015

Deng, X., Liang, S., Cai, X., Huang, S., Cheng, Z., Shi, Y., et al. (2019). Yolkshell structured Au nanostar@Metal-organic framework for synergistic chemophotothermal therapy in the second near-infrared window. Nano Lett. 19, 6772-6780. doi: 10.1021/acs.nanolett.9b01716

Dhada, K. S., Hernandez, D. S., and Suggs, L. J. (2019). In vivo photoacoustic tracking of mesenchymal stem cell viability. ACS Nano 13, 7791-7799. doi: 10.1021/acsnano.9b01802

Ding, F., Zhan, Y., Lu, X., and Sun, Y. (2018). Recent advances in near-infrared II fluorophores for multifunctional biomedical imaging. Chem. Sci. 9, 4370-4380. doi: $10.1039 / \mathrm{c} 8 \mathrm{sc} 01153 \mathrm{~b}$
Ding, S. Y., You, E. M., Tian, Z. Q., and Moskovits, M. (2017). Electromagnetic theories of surface-enhanced Raman spectroscopy. Chem. Soc. Rev. 46, 40424076. doi: $10.1039 / \mathrm{c} 7 \mathrm{cs} 00238 \mathrm{f}$

Ding, Y., Sun, Z., Tong, Z., Zhang, S., Min, J., Xu, Q., et al. (2020). Tumor microenvironment-responsive multifunctional peptide coated ultrasmall gold nanoparticles and their application in cancer radiotherapy. Theranostics 10, 5195-5208. doi: 10.7150/thno.45017

Dolmans, D. E., Fukumura, D., and Jain, R. K. (2003). Photodynamic therapy for cancer. Nat. Rev. Cancer 3, 380-387. doi: 10.1038/nrc1071

D'Souza, A. A., and Shegokar, R. (2016). Polyethylene glycol (PEG): a versatile polymer for pharmaceutical applications. Expert Opin. Drug Deliv. 13, 12571275. doi: 10.1080/17425247.2016.1182485

Dumani, D. S., Sun, I. C., and Emelianov, S. Y. (2019). Ultrasound-guided immunofunctional photoacoustic imaging for diagnosis of lymph node metastases. Nanoscale 11, 11649-11659. doi: 10.1039/c9nr02920f

Durr, N. J., Larson, T., Smith, D. K., Korgel, B. A., Sokolov, K., and Ben-Yakar, A. (2007). Two-photon luminescence imaging of cancer cells using molecularly targeted gold nanorods. Nano Lett. 7, 941-945. doi: 10.1021/nl062962v

Elci, S. G., Jiang, Y., Yan, B., Kim, S. T., Saha, K., Moyano, D. F., et al. (2016). Surface charge controls the suborgan biodistributions of gold nanoparticles. ACS Nano 10, 5536-5542. doi: 10.1021/acsnano.6b02086

Espinosa, A., Silva, A. K., Sanchez-Iglesias, A., Grzelczak, M., Pechoux, C., Desboeufs, K., et al. (2016). Cancer cell internalization of gold nanostars impacts their photothermal efficiency in vitro and in vivo: toward a plasmonic thermal fingerprint in tumoral environment. Adv. Healthc. Mater. 5, 10401048. doi: 10.1002/adhm.201501035

Feliu, N., Docter, D., Heine, M., Del Pino, P., Ashraf, S., Kolosnjaj-Tabi, J., et al. (2016). In vivo degeneration and the fate of inorganic nanoparticles. Chem. Soc. Rev. 45, 2440-2457. doi: 10.1039/c5cs00699f

Fischer, H. C., Hauck, T. S., Gomez-Aristizabal, A., and Chan, W. C. (2010). Exploring primary liver macrophages for studying quantum dot interactions with biological systems. Adv. Mater. 22, 2520-2524. doi: 10.1002/adma. 200904231

Fraga, S., Brandao, A., Soares, M. E., Morais, T., Duarte, J. A., Pereira, L., et al. (2014). Short- and long-term distribution and toxicity of gold nanoparticles in the rat after a single-dose intravenous administration. Nanomedicine 10, 1757-1766. doi: 10.1016/j.nano.2014.06.005

Fu, Q., Zhu, R., Song, J., Yang, H., and Chen, X. (2019). Photoacoustic imaging: contrast agents and their biomedical applications. Adv. Mater. 31:e1805875. doi: 10.1002/adma.201805875

Gamaleia, N. F., and Shton, I. O. (2015). Gold mining for PDT: great expectations from tiny nanoparticles. Photodiagnosis. Photodyn. Ther. 12, 221-231. doi: 10. 1016/j.pdpdt.2015.03.002

Gao, G., Chen, R., He, M., Li, J., Li, J., Wang, L., et al. (2019). Gold nanoclusters for Parkinson's disease treatment. Biomaterials 194, 36-46. doi: 10.1016/j. biomaterials.2018.12.013

Gao, N., Chen, Y., Li, L., Guan, Z., Zhao, T., Zhou, N., et al. (2014). Shapedependent two-photon photoluminescence of single gold nanoparticles. J. Phys. Chem. C 118, 13904-13911. doi: 10.1021/jp502038v

Ghorbani, M., and Hamishehkar, H. (2017). Decoration of gold nanoparticles with thiolated $\mathrm{pH}$-responsive polymeric (PEG-b-p(2-dimethylamio ethyl methacrylate-co-itaconic acid) shell: A novel platform for targeting of anticancer agent. Mater. Sci. Eng. C Mater. Biol. Appl. 81, 561-570. doi: 10.1016/ j.msec.2017.08.021

Golombek, S. K., May, J. N., Theek, B., Appold, L., Drude, N., Kiessling, F., et al. (2018). Tumor targeting via EPR: strategies to enhance patient responses. $A d v$. Drug Deliv. Rev. 130, 17-38. doi: 10.1016/j.addr.2018.07.007

Goncalves, A. S. C., Rodrigues, C. F., Moreira, A. F., and Correia, I. J. (2020). Strategies to improve the photothermal capacity of gold-based nanomedicines. Acta Biomater. 116, 105-137. doi: 10.1016/j.actbio.2020.09.008

Han, R., Zhao, M., Wang, Z., Liu, H., Zhu, S., Huang, L., et al. (2020). Super-efficient in vivo two-photon photodynamic therapy with a gold nanocluster as a Type I photosensitizer. ACS Nano 14, 9532-9544. doi: 10.1021/acsnano.9b05169

Hatz, S., Lambert, J. D., and Ogilby, P. R. (2007). Measuring the lifetime of singlet oxygen in a single cell: addressing the issue of cell viability. Photochem. Photobiol. Sci. 6, 1106-1116. doi: 10.1039/b707313e

He, M. Q., Chen, S., Yao, K., Meng, J., Wang, K., Yu, Y. L., et al. (2020). Precisely tuning LSPR property via "Peptide-Encoded" morphological evolution of gold 
nanorods for quantitative visualization of enzyme activity. Anal. Chem. 92, 1395-1401. doi: 10.1021/acs.analchem.9b04573

Higaki, T., Li, Y., Zhao, S., Li, Q., Li, S., Du, X. S., et al. (2019). Atomically tailored gold nanoclusters for catalytic application. Angew. Chem. Int. Ed. Engl. 58, 8291-8302. doi: 10.1002/anie.201814156

Higbee-Dempsey, E. M., Amirshaghaghi, A., Case, M. J., Bouche, M., Kim, J., Cormode, D. P., et al. (2020). Biodegradable gold nanoclusters with improved excretion due to $\mathrm{pH}$-triggered hydrophobic-to-hydrophilic transition. J. Am. Chem. Soc. 142, 7783-7794. doi: 10.1021/jacs.9b13813

Ho, D., Kretzmann, J. A., Norret, M., Toshniwal, P., Veder, J. P., Jiang, H., et al. (2018). Intracellular speciation of gold nanorods alters the conformational dynamics of genomic DNA. Nat. Nanotechnol. 13, 1148-1153. doi: 10.1038/ s41565-018-0272-2

Huang, J. A., Mousavi, M. Z., Zhao, Y., Hubarevich, A., Omeis, F., Giovannini, G., et al. (2019). SERS discrimination of single DNA bases in single oligonucleotides by electro-plasmonic trapping. Nat. Commun. 10:5321. doi: 10.1038/s41467019-13242-x

Huckaby, J. T., and Lai, S. K. (2018). PEGylation for enhancing nanoparticle diffusion in mucus. Adv. Drug Deliv. Rev. 124, 125-139. doi: 10.1016/j.addr. 2017.08.010

Hui, J., Cao, Y., Zhang, Y., Kole, A., Wang, P., Yu, G., et al. (2017). Realtime intravascular photoacoustic-ultrasound imaging of lipid-laden plaque in human coronary artery at 16 frames per second. Sci. Rep. 7:1417. doi: 10.1038/ s41598-017-01649-9

Jackman, J. A., Rahim Ferhan, A., and Cho, N. J. (2017). Nanoplasmonic sensors for biointerfacial science. Chem. Soc. Rev. 46, 3615-3660. doi: 10.1039/c6cs00494f

Jain, A., Barve, A., Zhao, Z., Jin, W., and Cheng, K. (2017). Comparison of avidin, neutravidin, and streptavidin as nanocarriers for efficient siRNA delivery. Mol. Pharm. 14, 1517-1527. doi: 10.1021/acs.molpharmaceut.6b00933

Jeon, M., Halbert, M. V., Stephen, Z. R., and Zhang, M. (2020). Iron oxide nanoparticles as $\mathrm{T} 1$ contrast agents for magnetic resonance imaging: fundamentals, challenges, applications, and prospectives. Adv. Mater. 2:e1906539. doi: 10.1002/adma.201906539

Ji, Y., Jones, C., Baek, Y., Park, G. K., Kashiwagi, S., and Choi, H. S. (2020). Nearinfrared fluorescence imaging in immunotherapy. Adv. Drug Deliv. Rev. 167, 121-134. doi: 10.1016/j.addr.2020.06.012

Jia, J., Liu, G., Xu, W., Tian, X., Li, S., Han, F., et al. (2020). Fine-tuning the homometallic interface of au-on-au nanorods and their photothermal therapy in the NIR-II window. Angew. Chem. Int. Ed. Engl. 59, 14443-14448. doi: 10.1002/anie.202000474

Jia, X., Xu, W., Ye, Z., Wang, Y., Dong, Q., Wang, E., et al. (2020). Functionalized Graphene@Gold nanostar/lipid for pancreatic cancer gene and photothermal synergistic therapy under photoacoustic/photothermal imaging dual-modal guidance. Small 16:e2003707. doi: 10.1002/smll.202003707

Kanamala, M., Wilson, W. R., Yang, M., Palmer, B. D., and Wu, Z. (2016). Mechanisms and biomaterials in $\mathrm{pH}$-responsive tumour targeted drug delivery: a review. Biomaterials 85, 152-167. doi: 10.1016/j.biomaterials.2016.01.061

Kang, H., Buchman, J. T., Rodriguez, R. S., Ring, H. L., He, J., Bantz, K. C., et al. (2019). Stabilization of Silver and Gold Nanoparticles: preservation and improvement of plasmonic functionalities. Chem. Rev. 119, 664-699. doi: 10. 1021/acs.chemrev.8b00341

Kenry, Duan, Y., and Liu, B. (2018). Recent advances of optical imaging in the second near-infrared window. Adv. Mater. 30:e1802394. doi: 10.1002/adma. 201802394

Kharazian, B., Lohse, S. E., Ghasemi, F., Raoufi, M., Saei, A. A., Hashemi, F., et al. (2018). Bare surface of gold nanoparticle induces inflammation through unfolding of plasma fibrinogen. Sci. Rep. 8:12557. doi: 10.1038/s41598-01830915-7

Kim, J., Lee, Y. M., Kang, Y., and Kim, W. J. (2014). Tumor-homing, size-tunable clustered nanoparticles for anticancer therapeutics. ACS Nano 8, 9358-9367. doi: $10.1021 / \mathrm{nn} 503349 \mathrm{~g}$

Kim, T., Lee, N., Arifin, D. R., Shats, I., Janowski, M., Walczak, P., et al. (2017). In vivo micro-CT imaging of human mesenchymal stem cells labeled with GoldPoly-L-lysine nanocomplexes. Adv. Funct. Mater. 27:1604213. doi: 10.1002/ adfm.201604213

Kreyling, W. G., Abdelmonem, A. M., Ali, Z., Alves, F., Geiser, M., Haberl, N., et al. (2015). In vivo integrity of polymer-coated gold nanoparticles. Nat. Nanotechnol. 10, 619-623. doi: 10.1038/nnano.2015.111
Kwon, K. C., Jo, E., Kwon, Y. W., Lee, B., Ryu, J. H., Lee, E. J., et al. (2017). Superparamagnetic gold nanoparticles synthesized on protein particle scaffolds for cancer theragnosis. Adv. Mater. 29:201701146. doi: 10.1002/adma. 201701146

Lee, N., Choi, S. H., and Hyeon, T. (2013). Nano-sized CT contrast agents. Adv. Mater. 25, 2641-2660. doi: 10.1002/adma.201300081

Lepeltier, E., Rijo, P., Rizzolio, F., Popovtzer, R., Petrikaite, V., Assaraf, Y. G., et al. (2020). Nanomedicine to target multidrug resistant tumors. Drug Resist Updat. 52:100704. doi: 10.1016/j.drup.2020.100704

Li, D., Liu, Q., Qi, Q., Shi, H., Hsu, E. C., Chen, W., et al. (2020a). Gold Nanoclusters for NIR-II Fluorescence Imaging of Bones. Small 16:e2003851. doi: 10.1002/ smll.202003851

Li, D., Yu, H., Guo, Z., Li, S., Li, Y., Guo, Y., et al. (2020b). SERS analysis of carcinoma-associated fibroblasts in a tumor microenvironment based on targeted 2D nanosheets. Nanoscale 12, 2133-2141. doi: 10.1039/c9nr08754k

Li, L., Xing, H., Zhang, J., and Lu, Y. (2019). Functional DNA molecules enable selective and stimuli-responsive nanoparticles for biomedical applications. Acc. Chem. Res. 52, 2415-2426. doi: 10.1021/acs.accounts.9b00167

Li, Q., Huang, C., Liu, L., Hu, R., and Qu, J. (2018). Effect of surface coating of gold nanoparticles on cytotoxicity and cell cycle progression. Nanomaterials (Basel) 8:8121063. doi: 10.3390/nano8121063

Li, S., Shen, X., Xu, Q. H., and Cao, Y. (2019). Gold nanorod enhanced conjugated polymer/photosensitizer composite nanoparticles for simultaneous two-photon excitation fluorescence imaging and photodynamic therapy. Nanoscale 11, 19551-19560. doi: 10.1039/c9nr05488j

Liebel, M., Pazos-Perez, N., van Hulst, N. F., and Alvarez-Puebla, R. A. (2020). Surface-enhanced Raman scattering holography. Nat. Nanotechnol. 15, 10051011. doi: 10.1038/s41565-020-0771-9

Lindquist, N. C., de Albuquerque, C. D. L., Sobral-Filho, R. G., Paci, I., and Brolo, A. G. (2019). High-speed imaging of surface-enhanced Raman scattering fluctuations from individual nanoparticles. Nat. Nanotechnol. 14, 981-987. doi: 10.1038/s41565-019-0535-6

Lipka, J., Semmler-Behnke, M., Sperling, R. A., Wenk, A., Takenaka, S., Schleh, C., et al. (2010). Biodistribution of PEG-modified gold nanoparticles following intratracheal instillation and intravenous injection. Biomaterials 31, 6574-6581. doi: 10.1016/j.biomaterials.2010.05.009

Liu, J., Huang, Y., Kumar, A., Tan, A., Jin, S., Mozhi, A., et al. (2014). pH-sensitive nano-systems for drug delivery in cancer therapy. Biotechnol. Adv. 32, 693-710. doi: 10.1016/j.biotechadv.2013.11.009

Liu, N., and Liedl, T. (2018). DNA-assembled advanced plasmonic architectures. Chem. Rev. 118, 3032-3053. doi: 10.1021/acs.chemrev.7b00225

Liu, Y. L., Zhu, J., Weng, G. J., Li, J. J., and Zhao, J. W. (2020). Gold nanotubes: synthesis, properties and biomedical applications. Mikrochim. Acta 187:612. doi: 10.1007/s00604-020-04460-y

Love, J. C., Estroff, L. A., Kriebel, J. K., Nuzzo, R. G., and Whitesides, G. M. (2005). Self-assembled monolayers of thiolates on metals as a form of nanotechnology. Chem. Rev. 105, 1103-1169. doi: 10.1021/cr0300789

Loynachan, C. N., Soleimany, A. P., Dudani, J. S., Lin, Y., Najer, A., Bekdemir, A., et al. (2019). Renal clearable catalytic gold nanoclusters for in vivo disease monitoring. Nat. Nanotechnol. 14, 883-890. doi: 10.1038/s41565-019-0527-6

Luo, C. H., Huang, C. T., Su, C. H., and Yeh, C. S. (2016). Bacteria-mediated hypoxia-specific delivery of nanoparticles for tumors imaging and therapy. Nano Lett. 16, 3493-3499. doi: 10.1021/acs.nanolett.6b00262

Luther, D. C., Huang, R., Jeon, T., Zhang, X., Lee, Y. W., Nagaraj, H., et al. (2020). Delivery of drugs, proteins, and nucleic acids using inorganic nanoparticles. Adv. Drug Deliv. Rev. 156, 188-213. doi: 10.1016/j.addr.2020.06.020

Ma, N., Wu, F. G., Zhang, X., Jiang, Y. W., Jia, H. R., Wang, H. Y., et al. (2017). Shape-dependent radiosensitization effect of gold nanostructures in cancer radiotherapy: comparison of gold nanoparticles, nanospikes, and nanorods. ACS Appl. Mater. Interf. 9, 13037-13048. doi: 10.1021/acsami.7b0 1112

Ma, X., Song, S., Kim, S., Kwon, M. S., Lee, H., Park, W., et al. (2019). Single gold-bridged nanoprobes for identification of single point DNA mutations. Nat. Commun. 10:836. doi: 10.1038/s41467-019-08769-y

Mangadlao, J. D., Wang, X., McCleese, C., Escamilla, M., Ramamurthy, G., Wang, Z., et al. (2018). Prostate-specific membrane antigen targeted gold nanoparticles for theranostics of prostate cancer. ACS Nano. 12, 3714-3725. doi: 10.1021/ acsnano.8b00940 
McDonald, P. C., Chafe, S. C., Brown, W. S., Saberi, S., Swayampakula, M., Venkateswaran, G., et al. (2019). Regulation of $\mathrm{pH}$ by carbonic anhydrase 9 mediates survival of pancreatic cancer cells with activated KRAS in response to hypoxia. Gastroenterology 157, 823-837. doi: 10.1053/j.gastro.2019.05.004

Mejia-Salazar, J. R., and Oliveira, O. N. Jr. (2018). Plasmonic biosensing. Chem. Rev. 118, 10617-10625. doi: 10.1021/acs.chemrev.8b00359

Meng, Z., Zhou, X., She, J., Zhang, Y., Feng, L., and Liu, Z. (2019). Ultrasoundresponsive conversion of microbubbles to nanoparticles to enable backgroundfree in vivo photoacoustic imaging. Nano. Lett. 19, 8109-8117. doi: 10.1021/acs. nanolett.9b03331

Mousavi, S. M., Zarei, M., Hashemi, S. A., Ramakrishna, S., Chiang, W. H., Lai, C. W., et al. (2020). Gold nanostars-diagnosis, bioimaging and biomedical applications. Drug Metab. Rev. 52, 299-318. doi: 10.1080/03602532.2020. 1734021

Moy, A. J., and Tunnell, J. W. (2017). Combinatorial immunotherapy and nanoparticle mediated hyperthermia. Adv. Drug Deliv. Rev. 114, 175-183. doi: 10.1016/j.addr.2017.06.008

Nel, A., Ruoslahti, E., and Meng, H. (2017). New insights into "Permeability" as in the enhanced permeability and retention effect of cancer nanotherapeutics. ACS Nano 11, 9567-9569. doi: 10.1021/acsnano.7b07214

Nicolson, F., Ali, A., Kircher, M. F., and Pal, S. (2020). DNA nanostructures and DNA-functionalized nanoparticles for cancer theranostics. Adv. Sci. (Weinh) 7:2001669. doi: 10.1002/advs.202001669

Ogunyankin, M. O., Shin, J. E., Lapotko, D. O., Ferry, V. E., and Zasadzinski, J. A. (2018). Optimizing the NIR fluence threshold for nanobubble generation by controlled synthesis of $10-40 \mathrm{~nm}$ hollow gold nanoshells. Adv. Funct. Mater. 28:201705272. doi: 10.1002/adfm.201705272

Pan, Y., Neuss, S., Leifert, A., Fischler, M., Wen, F., Simon, U., et al. (2007). Sizedependent cytotoxicity of gold nanoparticles. Small 3, 1941-1949. doi: 10.1002/ smll.200700378

Peng, J., Yang, Q., Shi, K., Xiao, Y., Wei, X., and Qian, Z. (2019). Intratumoral fate of functional nanoparticles in response to microenvironment factor: Implications on cancer diagnosis and therapy. Adv. Drug Deliv. Rev. 143, 37-67. doi: 10.1016/ j.addr.2019.06.007

Piao, J. G., Wang, L., Gao, F., You, Y. Z., Xiong, Y., and Yang, L. (2014). Erythrocyte membrane is an alternative coating to polyethylene glycol for prolonging the circulation lifetime of gold nanocages for photothermal therapy. ACS Nano. 8, 10414-10425. doi: 10.1021/nn503779d

Poderys, V., Jarockyte, G., Bagdonas, S., Karabanovas, V., and Rotomskis, R. (2020). Protein-stabilized gold nanoclusters for PDT: ROS and singlet oxygen generation. J. Photochem. Photobiol. B 204, 111802. doi: 10.1016/j.jphotobiol. 2020.111802

Puppulin, L., Hosogi, S., Sun, H., Matsuo, K., Inui, T., Kumamoto, Y., et al. (2018). Bioconjugation strategy for cell surface labelling with gold nanostructures designed for highly localized $\mathrm{pH}$ measurement. Nat. Commun. 9:5278. doi: 10.1038/s41467-018-07726-5

Qiao, X., Su, B., Liu, C., Song, Q., Luo, D., Mo, G., et al. (2018). Selective surface enhanced raman scattering for quantitative detection of lung cancer biomarkers in superparticle@MOF structure. Adv. Mater. 30:1702275. doi: 10.1002/adma. 201702275

Qiu, K., Du, Y., Liu, J., Guan, J. L., Chao, H., and Diao, J. (2020). Super-resolution observation of lysosomal dynamics with fluorescent gold nanoparticles. Theranostics 10, 6072-6081. doi: 10.7150/thno.42134

Rafiei, P., and Haddadi, A. (2017). Docetaxel-loaded PLGA and PLGAPEG nanoparticles for intravenous application: pharmacokinetics and biodistribution profile. Int. J. Nanomed. 12, 935-947. doi: 10.2147/IJN.S121881

Ramos, D., Malvar, O., Davis, Z. J., Tamayo, J., and Calleja, M. (2018). Nanomechanical plasmon Spectroscopy of single gold nanoparticles. Nano. Lett. 18, 7165-7170. doi: 10.1021/acs.nanolett.8b03236

Rebello Sousa Dias, M., and Leite, M. S. (2019). Alloying: a platform for metallic materials with on-demand optical response. Acc. Chem. Res. 52, 2881-2891. doi: 10.1021/acs.accounts.9b00153

Rengan, A. K., Bukhari, A. B., Pradhan, A., Malhotra, R., Banerjee, R., Srivastava, R., et al. (2015). In vivo analysis of biodegradable liposome gold nanoparticles as efficient agents for photothermal therapy of cancer. Nano. Lett. 15, 842-848. doi: $10.1021 / \mathrm{nl} 5045378$
Riley, R. S., and Day, E. S. (2017). Gold nanoparticle-mediated photothermal therapy: applications and opportunities for multimodal cancer treatment. WIREs Nanomed. Nanobiotechnol. 9:e1449. doi: 10.1002/wnan.1449

Sabale, S., Kandesar, P., Jadhav, V., Komorek, R., Motkuri, R. K., and Yu, X. Y. (2017). Recent developments in the synthesis, properties, and biomedical applications of core/shell superparamagnetic iron oxide nanoparticles with gold. Biomater. Sci. 5, 2212-2225. doi: 10.1039/c7bm00723j

Samanta, A., Jana, S., Das, R. K., and Chang, Y. T. (2014). Biocompatible surface-enhanced Raman scattering nanotags for in vivo cancer detection. Nanomedicine (Lond.) 9, 523-535. doi: 10.2217/nnm.13.222

Schon, J. H., Meng, H., and Bao, Z. (2003). Retraction: self-assembled monolayer organic field-effect transistors. Nature 422:92. doi: 10.1038/nature01464

Shanmugam, V., Selvakumar, S., and Yeh, C. S. (2014). Near-infrared lightresponsive nanomaterials in cancer therapeutics. Chem. Soc. Rev. 43, 62546287. doi: $10.1039 / \mathrm{c} 4 \mathrm{cs} 00011 \mathrm{k}$

Sharifi, M., Attar, F., Saboury, A. A., Akhtari, K., Hooshmand, N., Hasan, A., et al. (2019). Plasmonic gold nanoparticles: optical manipulation, imaging, drug delivery and therapy. J. Control Release 31, 170-189. doi: 10.1016/j.jconrel.2019. 08.032

Shen, Y., Shuhendler, A. J., Ye, D., Xu, J. J., and Chen, H. Y. (2016). Twophoton excitation nanoparticles for photodynamic therapy. Chem. Soc. Rev. 45, 6725-6741. doi: 10.1039/c6cs00442c

Shi, H., Wang, Z., Huang, C., Gu, X., Jia, T., Zhang, A., et al. (2016). A functional CT Contrast agent for in vivo imaging of tumor hypoxia. Small 12, 3995-4006. doi: 10.1002/smll.201601029

Shi, X., Perry, H. L., and Wilton-Ely, J. (2021). Strategies for the functionalisation of gold nanorods to reduce toxicity and aid clinical translation. Nanotheranostics 5, 155-165. doi: 10.7150/ntno.56432

Shin, H., Oh, S., Kang, D., and Choi, Y. (2020). Protein quantification and imaging by surface-enhanced raman spectroscopy and similarity analysis. Adv. Sci. (Weinh.) 7:1903638. doi: 10.1002/advs.201903638

Sies, H., and Jones, D. P. (2020). Reactive oxygen species (ROS) as pleiotropic physiological signalling agents. Nat. Rev. Mol. Cell Biol. 21, 363-383. doi: 10. 1038/s41580-020-0230-3

Sindhwani, S., Syed, A. M., Ngai, J., Kingston, B. R., Maiorino, L., Rothschild, J., et al. (2020). The entry of nanoparticles into solid tumours. Nat. Mater. 19, 566-575. doi: 10.1038/s41563-019-0566-2

Smith, A. M., Mancini, M. C., and Nie, S. (2009). Bioimaging: second window for in vivo imaging. Nat. Nanotechnol. 4, 710-711. doi: 10.1038/nnano.2009.326

Sun, Q., Shi, X., Feng, J., Zhang, Q., Ao, Z., Ji, Y., et al. (2018). Cytotoxicity and cellular responses of gold nanorods to smooth muscle cells dependent on surface chemistry coupled action. Small 14:e1803715. doi: 10.1002/smll. 201803715

Takano, S., Hasegawa, S., Suyama, M., and Tsukuda, T. (2018). Hydride doping of chemically modified gold-based superatoms. Acc. Chem. Res. 51, 3074-3083. doi: 10.1021/acs.accounts.8b00399

Talamini, L., Violatto, M. B., Cai, Q., Monopoli, M. P., Kantner, K., Krpetic, Z., et al. (2017). Influence of size and shape on the anatomical distribution of endotoxin-free gold nanoparticles. ACS Nano. 11, 5519-5529. doi: 10.1021/ acsnano.7b00497

Tan, S. F., Raj, S., Bisht, G., Annadata, H. V., Nijhuis, C. A., Kral, P., et al. (2018). Nanoparticle interactions guided by shape-dependent hydrophobic forces. $A d v$. Mater. 30:e1707077. doi: 10.1002/adma.201707077

Tang, Y., Shi, H., Cheng, D., Zhang, J., Lin, Y., Xu, Y., et al. (2019). pH-Activatable tumor-targeting gold nanoprobe for near-infrared fluorescence/CT dual-modal imaging in vivo. Colloid. Surf B Biointerfac. 179, 56-65. doi: 10.1016/j.colsurfb. 2019.03.049

Tebbe, M., Kuttner, C., Mannel, M., Fery, A., and Chanana, M. (2015). Colloidally stable and surfactant-free protein-coated gold nanorods in biological media. ACS Appl. Mater. Interfac. 7, 5984-5991. doi: 10.1021/acsami.5b00335

Venkatachalam, M. A., and Rennke, H. G. (1978). The structural and molecular basis of glomerular filtration. Circ. Res. 43, 337-347. doi: 10.1161/01.res.43.3. 337

Vidal, C., Tomas-Gamasa, M., Destito, P., Lopez, F., and Mascarenas, J. L. (2018). Concurrent and orthogonal gold(I) and ruthenium(II) catalysis inside living cells. Nat. Commun. 9:1913. doi: 10.1038/s41467-018-04314-5 
Vigderman, L., Khanal, B. P., and Zubarev, E. R. (2012). Functional gold nanorods: synthesis, self-assembly, and sensing applications. Adv. Mater. 24:5014. doi: 10.1002/adma.201201690

Viswambari Devi, R., Doble, M., and Verma, R. S. (2015). Nanomaterials for early detection of cancer biomarker with special emphasis on gold nanoparticles in immunoassays/sensors. Biosens. Bioelectron. 68, 688-698. doi: 10.1016/j.bios. 2015.01.066

Vo-Dinh, T., Fales, A. M., Griffin, G. D., Khoury, C. G., Liu, Y., Ngo, H., et al. (2013). Plasmonic nanoprobes: from chemical sensing to medical diagnostics and therapy. Nanoscale 5, 10127-10140. doi: 10.1039/c3nr03633b

Wang, C., Wang, Y., Zhang, L., Miron, R. J., Liang, J., Shi, M., et al. (2018). Pretreated macrophage-membrane-coated gold nanocages for precise drug delivery for treatment of bacterial infections. Adv. Mater. 30:e1804023. doi: 10.1002/adma.201804023

Wang, L., Yuan, Y., Lin, S., Huang, J., Dai, J., Jiang, Q., et al. (2016). Photothermo-chemotherapy of cancer employing drug leakage-free gold nanoshells. Biomaterials 78, 40-49. doi: 10.1016/j.biomaterials.2015.11.024

Wang, Z., Zong, S., Wang, Y., Li, N., Li, L., Lu, J., et al. (2018). Screening and multiple detection of cancer exosomes using an SERS-based method. Nanoscale 10, 9053-9062. doi: 10.1039/c7nr09162a

Wilhelm, C., Gazeau, F., and Silva, A. K. A. (2019). Physically-triggered nanosystems for therapy and diagnosis. Adv. Drug Deliv. Rev. 138, 1-2. doi: 10.1016/j.addr.2019.03.001

Willets, K. A., Wilson, A. J., Sundaresan, V., and Joshi, P. B. (2017). Superresolution imaging and plasmonics. Chem. Rev. 117, 7538-7582. doi: 10.1021/ acs.chemrev.6b00547

Wilson, A. J., Devasia, D., and Jain, P. K. (2020). Nanoscale optical imaging in chemistry. Chem. Soc. Rev. 49, 6087-6112. doi: 10.1039/d0cs00338g

Wozniak, A., Malankowska, A., Nowaczyk, G., Grzeskowiak, B. F., Tusnio, K., Slomski, R., et al. (2017). Size and shape-dependent cytotoxicity profile of gold nanoparticles for biomedical applications. J. Mater. Sci. Mater. Med. 28:92. doi: 10.1007/s10856-017-5902-y

Wu, J., Wang, X., Wang, Q., Lou, Z., Li, S., Zhu, Y., et al. (2019). Nanomaterials with enzyme-like characteristics (nanozymes): next-generation artificial enzymes (II). Chem. Soc. Rev. 48, 1004-1076. doi: 10.1039/c8cs00457a

Xia, Y., Li, W., Cobley, C. M., Chen, J., Xia, X., Zhang, Q., et al. (2011). Gold nanocages: from synthesis to theranostic applications. Acc. Chem. Res. 44, 914-924. doi: 10.1021/ar200061q

Xiao, W., Ruan, S., Yu, W., Wang, R., Hu, C., Liu, R., et al. (2017). Normalizing tumor vessels to increase the enzyme-induced retention and targeting of gold nanoparticle for breast cancer imaging and treatment. Mol. Pharm. 14, 34893498. doi: 10.1021/acs.molpharmaceut.7b00475

Yang, L., Zhou, Z., Song, J., and Chen, X. (2019). Anisotropic nanomaterials for shape-dependent physicochemical and biomedical applications. Chem. Soc. Rev. 48, 5140-5176. doi: 10.1039/c9cs00011a

Yhee, J. Y., Jeon, S., Yoon, H. Y., Shim, M. K., Ko, H., Min, J., et al. (2017). Effects of tumor microenvironments on targeted delivery of glycol chitosan nanoparticles. J. Control Release 267, 223-231. doi: 10.1016/j.jconrel.2017.09. 015

You, Q., Zhang, K., Liu, J., Liu, C., Wang, H., Wang, M., et al. (2020). Persistent Regulation of tumor hypoxia microenvironment via a bioinspired Pt-based oxygen nanogenerator for multimodal imaging-guided synergistic phototherapy. Adv. Sci. (Weinh) 7:1903341. doi: 10.1002/advs.201903341

Yu, W., He, X., Yang, Z., Yang, X., Xiao, W., Liu, R., et al. (2019). Sequentially responsive biomimetic nanoparticles with optimal size in combination with checkpoint blockade for cascade synergetic treatment of breast cancer and lung metastasis. Biomaterials 217:119309.

Zhang, J., Liang, L., Guan, X., Deng, R., Qu, H., Huang, D., et al. (2018). In situ, accurate, surface-enhanced Raman scattering detection of cancer cell nucleus with synchronous location by an alkyne-labeled biomolecular probe. Anal. Bioanal. Chem. 410, 585-594. doi: 10.1007/s00216-017-0761-4

Zhang, L., Wan, S. S., Li, C. X., Xu, L., Cheng, H., and Zhang, X. Z. (2018). An Adenosine triphosphate-responsive autocatalytic fenton nanoparticle for tumor ablation with self-supplied $\mathrm{H} 2 \mathrm{O} 2$ and acceleration of $\mathrm{Fe}(\mathrm{III}) / \mathrm{Fe}$ (II) conversion. Nano. Lett. 18, 7609-7618. doi: 10.1021/acs.nanolett.8b03178

Zhang, Q., Yang, M., Zhu, Y., and Mao, C. (2018). Metallic nanoclusters for cancer imaging and therapy. Curr. Med. Chem. 25, 1379-1396. doi: 10.2174/ 0929867324666170331122757
Zhang, T. T., Xu, C. H., Zhao, W., Gu, Y., Li, X. L., Xu, J. J., et al. (2018). A redox-activated theranostic nanoagent: toward multi-mode imaging guided chemo-photothermal therapy. Chem. Sci. 9, 6749-6757. doi: 10.1039/c8sc0 $2446 \mathrm{~d}$

Zhang, W., Caldarola, M., Lu, X., and Orrit, M. (2018). Plasmonic enhancement of two-photon-excited luminescence of single quantum dots by individual gold nanorods. ACS Photonics 5, 2960-2968. doi: 10.1021/acsphotonics.8b 00306

Zhang, X. D., Luo, Z., Chen, J., Song, S., Yuan, X., Shen, X., et al. (2015). Ultrasmall glutathione-protected gold nanoclusters as next generation radiotherapy sensitizers with high tumor uptake and high renal clearance. Sci. Rep. 5:8669. doi: $10.1038 /$ srep08669

Zhang, X. D., Wu, D., Shen, X., Liu, P. X., Yang, N., Zhao, B., et al. (2011). Sizedependent in vivo toxicity of PEG-coated gold nanoparticles. Int. J. Nanomed. 6, 2071-2081. doi: 10.2147/IJN.S21657

Zhang, X., Xi, Z., Machuki, J. O., Luo, J., Yang, D., Li, J., et al. (2019). Gold cube-in-cube based oxygen nanogenerator: a theranostic nanoplatform for modulating tumor microenvironment for precise chemo-phototherapy and multimodal imaging. ACS Nano. 13, 5306-5325. doi: 10.1021/acsnano.8b0 9786

Zhang, Y., Elechalawar, C. K., Hossen, M. N., Francek, E. R., Dey, A., Wilhelm, S., et al. (2021). Gold nanoparticles inhibit activation of cancer-associated fibroblasts by disrupting communication from tumor and microenvironmental cells. Bioact. Mater. 6, 326-332. doi: 10.1016/j.bioactmat.2020.08.009

Zhao, J. Y., Cui, R., Zhang, Z. L., Zhang, M., Xie, Z. X., and Pang, D. W. (2014). Cytotoxicity of nucleus-targeting fluorescent gold nanoclusters. Nanoscale 6, 13126-13134. doi: 10.1039/c4nr04227a

Zhao, J., Qin, Z., Wu, J., Li, L., Jin, Q., and Ji, J. (2017). Zwitterionic stealth peptide-protected gold nanoparticles enable long circulation without the accelerated blood clearance phenomenon. Biomater. Sci. 6, 200-206. doi: 10. 1039/c7bm00747g

Zhou, W., Gao, X., Liu, D., and Chen, X. (2015). Gold nanoparticles for in vitro diagnostics. Chem. Rev. 115, 10575-10636. doi: 10.1021/acs.chemrev.5b0 0100

Zhou, Z., Song, J., Nie, L., and Chen, X. (2016). Reactive oxygen species generating systems meeting challenges of photodynamic cancer therapy. Chem. Soc. Rev. 45, 6597-6626. doi: 10.1039/c6cs00271d

Zhou, Z., Yang, L., Gao, J., and Chen, X. (2019). Structure-relaxivity relationships of magnetic nanoparticles for magnetic resonance imaging. Adv. Mater. 31:e1804567. doi: 10.1002/adma.201804567

Zhu, R., Su, L., Dai, J., Li, Z. W., Bai, S., Li, Q., et al. (2020). Biologically responsive plasmonic assemblies for second near-infrared window photoacoustic imagingguided concurrent chemo-immunotherapy. ACS Nano. 14, 3991-4006. doi: 10.1021/acsnano.9b07984

Zhu, S., Yung, B. C., Chandra, S., Niu, G., Antaris, A. L., and Chen, X. (2018). Nearinfrared-II (NIR-II) bioimaging via Off-Peak NIR-I fluorescence emission. Theranostics 8, 4141-4151. doi: 10.7150/thno.27995

Zhu, X., Sarwar, M., Zhu, J. J., Zhang, C., Kaushik, A., and Li, C. Z. (2019). Using a glucose meter to quantitatively detect disease biomarkers through a universal nanozyme integrated lateral fluidic sensing platform. Biosens. Bioelectron. 126, 690-696. doi: 10.1016/j.bios.2018.11.033

Zong, C., Xu, M., Xu, L. J., Wei, T., Ma, X., Zheng, X. S., et al. (2018). Surfaceenhanced raman spectroscopy for bioanalysis: reliability and challenges. Chem. Rev. 118, 4946-4980. doi: 10.1021/acs.chemrev.7b00668

Conflict of Interest: The authors declare that the research was conducted in the absence of any commercial or financial relationships that could be construed as a potential conflict of interest.

The handling editor declared a past co-authorship with one of the authors, $\mathrm{HZ}$

Copyright (c) 2021 Gao, Zhang, Gao, Zhang, Zhu and Wang. This is an openaccess article distributed under the terms of the Creative Commons Attribution License (CC BY). The use, distribution or reproduction in other forums is permitted, provided the original author(s) and the copyright owner(s) are credited and that the original publication in this journal is cited, in accordance with accepted academic practice. No use, distribution or reproduction is permitted which does not comply with these terms. 